\title{
Changing supply rates for input-output to state stable discrete-time nonlinear systems with applications
}

\author{
Dina Shona Laila ${ }^{1,2}$ and Dragan Nešić ${ }^{1}$
}

\begin{abstract}
We present results on changing supply rates for input-output to state stable (IOSS) discrete-time nonlinear systems. Our results can be used to combine two Lyapunov functions, none of which can be used to verify that the system has a certain property, into a new composite Lyapunov function from which the property of interest can be concluded. The results are stated for parameterized families of discrete-time systems that naturally arise when an approximate discrete-time model is used to design a controller for a sampled-data system. We present several applications of our results: (i) a LaSalle criterion for input to state stability (ISS) of discrete-time systems; (ii) constructing ISS Lyapunov functions for time-varying discrete-time cascaded systems; (iii) testing ISS of discrete-time systems using positive semidefinite Lyapunov functions; (iv) observer-based input to state stabilization of discrete-time systems. Our results are exploited in a case study of a two link manipulator and some simulation results that illustrate advantages of our approach are presented.
\end{abstract}

Key words: Discrete-time; Input-to-state stability; Lyapunov method; Nonlinear control; Supply rates.

\section{Introduction}

The Lyapunov method is one of the most important and useful methods in stability analysis and design of nonlinear control systems (see $[15,16,28]$ ). A very useful method for a partial construction of Lyapunov functions was discussed in [31] where it was shown how it is possible to combine two Lyapunov functions, none of which can be used to conclude a property of interest, into a new composite Lyapunov function from which the desired property follows. Results in [31] apply to the analysis of input to state stability (ISS) property of continuoustime cascade-connected systems. In [1] a similar proof technique was used to combine a Lyapunov function whose derivative is negative semidefinite and another Lyapunov function that characterizes a detectability property, which is called input-output to state stability (IOSS) (see [32]), into a new Lyapunov function from which ISS of a continuous-time system follows. A discrete-time counterpart of results in [31] was presented in [26]. These results and proof techniques were used in discrete-time backstepping [25], stability of continuoustime cascades [3,31], stability of discrete-time cascades [26], continuous-time stabilization of robot manipu-

\footnotetext{
1 The authors are with the Department of Electrical and Electronic Engineering, The University of Melbourne, Parkville, 3010, Victoria, Australia.

2 Corresponding author. Phone:+61-3-83449194, Fax:+613-83446678, E-mail: \{dsl, d.nesic\}@ee.mu.oz.au.
}

lators [1] and $L_{p}$ stability of time-varying nonlinear sampled-data systems [37]. A related Lyapunov based method for interconnected ISS continuous-time systems satisfying a small-gain condition can be found in [11].

The main purpose of this paper is to extend the results from $[1,26,31]$ so that they apply to families of discretetime systems parameterized by a positive parameter (sampling period). We consider a particular type of semiglobal practical stability properties of the parameterized discrete-time systems that arise naturally when approximate discrete-time models are used to design controllers for sampled-data nonlinear systems. The stability properties we consider depend in a very particular manner on the parameter and, in particular, they are not uniform in the parameter. Motivation for our approach is presented in the next section and more information can be found in [22-25,27].

Another important contribution of our work is that we present a unifying framework that allows us to consider a range of seemingly unrelated results in a unified manner. We are not aware of any similar unification for continuous-time systems and in this sense our approach may have important implications even in continuoustime. Our main results immediately apply to: (i) a LaSalle criterion for ISS of discrete-time systems (see also [1]); (ii) constructing ISS Lyapunov functions for time-varying discrete-time cascade-connected systems (see also $[10,12,26,31]$ ); (iii) testing ISS of discrete-time 
systems using positive semidefinite Lyapunov functions (see also $[5,9]$ ); (iv) observer-based input to state stabilization of discrete-time systems (see also $[13,14]$ ). We emphasize that our results have potential for further important applications and the case study presented at the end of the paper illustrates how some of our results may be used for sampled-data controller design. Main results of this paper, applications and case study are respectively presented in Section 4,5 and 6 . The proofs of main results are provided in the Appendix.

\section{Background and motivation}

Most control systems are nowadays sampled-data in nature. Indeed, the controller is usually implemented digitally using a computer and it is inter-connected with a continuous-time plant via $\mathrm{D} / \mathrm{A}$ and $\mathrm{A} / \mathrm{D}$ converters. Since it is in general impossible to compute the exact discrete-time model of a continuous-time nonlinear plant, approximate discrete-time models such as Euler, are often used for control design. This approach was taken, for instance, in $[6,8,19,29]$ for several special classes of systems. Recently, a general unified framework for controller design based on approximate discrete-time models was presented in $[23,27]$ for the stabilization problem and further generalized in [22] for the input to state stabilization problem and in [21] for integral input to state stabilization problem. Advantages of this approach were illustrated in [25] where it was shown that the Euler based backstepping controller may outperform the emulated backstepping controller.

It is the main purpose of the current paper to further contribute to the approach that was pursued in [21$23,27]$. In order to motivate stronger our contribution, we present a result from [22] on input to state stabilization via approximate discrete-time models that is also needed in Section 6. Our interest in input to state stabilization is motivated by numerous applications of this robust stability property that have appeared in the literature $[10,16,30,33]$.

Consider a continuous-time nonlinear plant ${ }^{3}$

$$
\dot{x}(t)=f(x(t), u(t), w(t)) ; \quad y(t)=h(x(t)),
$$

where $x \in \mathbb{R}^{n_{x}}, u \in \mathbb{R}^{m}, w \in \mathbb{R}^{p}$ and $y \in \mathbb{R}^{l}$ are respectively the state, control input, exogenous disturbance and output. We assume that for any given $x_{0}, u(\cdot)$ and $w(\cdot)$ the differential equation in (1) has a unique solution defined on its maximal interval of existence $\left[0, t_{\max }\right)$. This may be guaranteed, for instance, by requiring $f$ in (1) to be locally Lipschitz. The control is taken to be a piecewise constant signal $u(t)=u(k T)=: u(k), \forall t \in$

\footnotetext{
${ }^{3}$ For any unfamiliar notation, readers are referred to the next section.
}

$[k T,(k+1) T), k \in \mathbb{N}$, where $T>0$ is the sampling period. Suppose that the disturbance $w(\cdot)$ is constant during sampling intervals, that is $w(t)=w(k), \forall t \in$ $[k T,(k+1) T)$ (a more general situation when $w(\cdot)$ is an arbitrary measurable disturbance was considered in [22]). Also, we assume that some combination (output) or all of the states $(x(k):=x(k T))$ are available at sampling instant $k T, k \in \mathbb{N}$. The exact discrete-time model for the plant (1), which describes the plant behavior at sampling instants $k T$, is obtained by integrating the initial value problem

$$
\dot{x}(t)=f(x(t), u(k), w(t)),
$$

with given $w(k), u(k)$ and $x_{0}=x(k)$, over the sampling interval $[k T,(k+1) T]$. If we denote by $x(t)$ the solution of the initial value problem (2) at time $t$ with given $x_{0}=$ $x(k), u(k)$ and $w(k)$, then the exact discrete-time model of (1) can be written as:

$$
\begin{aligned}
x(k+1) & =x(k)+\int_{k T}^{(k+1) T} f(x(\tau), u(k), w(k)) d \tau \\
& =: F_{T}^{e}(x(k), u(k), w(k)) .
\end{aligned}
$$

We emphasize that $F_{T}^{e}$ is not known in most cases. Indeed, in order to compute $F_{T}^{e}$ we have to solve the initial value problem (2) analytically and this is usually impossible since $f$ in (1) is nonlinear. Hence, we will use an approximate discrete-time model of the plant to design a discrete-time controller for the original plant (1).

Different approximate discrete-time models can be obtained using different methods, such as a classical RungeKutta numerical integration scheme (such as Euler) for the initial value problem (2) [20,34]. The approximate discrete-time model can be written as

$$
x(k+1)=F_{T}^{a}(x(k), u(k), w(k)) .
$$

For instance, the Euler approximate model is $x(k+1)=$ $x(k)+T f(x(k), u(k), w(k))$. The sampling period $T$ is assumed to be a design parameter which can be arbitrarily assigned. Since we are dealing with a family of approximate discrete-time models $F_{T}^{a}$, parameterized by $T$, in order to achieve a certain objective we need in general to obtain a family of controllers, also parameterized by $T$. We consider a family of dynamic feedback controllers

$$
z(k+1)=G_{T}(x(k), z(k)) ; u(k)=u_{T}(x(k), z(k)),
$$

where $z \in \mathbb{R}^{n_{z}}$.

We emphasize that if the controller (5) input to state stabilizes the approximate model (4) for all small $T$, this does not guarantee that the same controller would approximately input to state stabilize the exact model (3) for all small $T$ (see $[4,7,27])$. 
The following result provides a framework for controller design via approximate discrete-time models:

Theorem 2.1 [22] Suppose that there exist $\underline{\alpha}, \bar{\alpha}, \alpha \in$ $\mathcal{K}_{\infty}$ and $\sigma \in \mathcal{K}$, and for any strictly positive real numbers $\left(\Delta_{1}, \Delta_{2}, \Delta_{3}, \nu\right)$ there exist $\varrho \in \mathcal{K}_{\infty}$, strictly positive real numbers $T^{*}, L, M$ such that for all $T \in\left(0, T^{*}\right)$ there exists a function $V_{T}: \mathbb{R}^{n_{x}+n_{z}} \rightarrow \mathbb{R}_{>0}$ such that for all $|(x, z)| \leq \Delta_{1},|u| \leq \Delta_{2},|w| \leq \Delta_{3}, T \in\left(0, T^{*}\right)$ we have:

- SP-ISS Lyapunov conditions for closed-loop approximate:

$$
\begin{gathered}
\underline{\alpha}(|(x, z)|) \leq V_{T}(x, z) \leq \bar{\alpha}(|(x, z)|) \\
V_{T}\left(F_{T}^{a}\left(x, u_{T}(x, z), w\right), G_{T}(x, z)\right)-V_{T}(x, z) \\
\leq T(-\alpha(|(x, z)|)+\sigma(|w|)+\nu)
\end{gathered}
$$

and, moreover, for all $T \in\left(0, T^{*}\right)$ and all $x_{1}, x_{2}, z$ with $\max \left\{\left|\left(x_{1}, z\right)\right|,\left|\left(x_{2}, z\right)\right|\right\} \leq \Delta_{1}$

$$
\left|V_{T}\left(x_{1}, z\right)-V_{T}\left(x_{2}, z\right)\right| \leq L\left|x_{1}-x_{2}\right| .
$$

- consistency between $F_{T}^{a}$ and $F_{T}^{e}$ :

$$
\left|F_{T}^{e}(x, u, w)-F_{T}^{a}(x, u, w)\right| \leq T \varrho(T) .
$$

- uniform local boundedness of $u_{T}$ :

$$
\left|u_{T}(x, z)\right| \leq M \text {. }
$$

Then, there exists $\beta \in \mathcal{K} L, \gamma \in \mathcal{G}$ such that for any strictly positive real numbers $\left(\widetilde{\Delta}_{1}, \widetilde{\Delta}_{2}, \widetilde{\nu}\right)$ there exists $\widetilde{T}>$ 0 such that for all $|(x(0), z(0))| \leq \widetilde{\Delta}_{1},\|w\|_{\infty} \leq \widetilde{\Delta}_{2}$ and $T \in(0, \widetilde{T})$ the solutions of (3), (5) satisfy:

- SP-ISS of closed-loop exact: $|(x(k), z(k))| \leq$ $\beta(|(x(0), z(0))|, k T)+\gamma\left(\|w\|_{\infty}\right)+\widetilde{\nu}, \quad \forall k \geq 0$.

We emphasize that the consistency condition in Theorem 2.1 is checkable although $F_{T}^{e}$ is not known in general. This condition is commonly used in numerical analysis literature [34]. The conditions (6),(7) of Theorem 2.1 are hard to check in general and one of the main contributions of the current paper is in presenting technical results that can be used to verify that conditions equivalent with (6),(7) hold for a family of parameterized discretetime systems. These technical conditions can be then used in conjunction with Theorem 2.1 to design input to state stabilizing controllers for sampled-data nonlinear plants via their approximate discrete-time models. This approach is illustrated in Section 6 where we consider input to state stabilization of a two link manipulator via its Euler approximate discrete-time model.

\section{Preliminaries}

The set of real and natural numbers (including 0) are denoted respectively by $\mathbb{R}$ and $\mathbb{N}$. $\mathcal{S N}$ denotes the class of smooth nondecreasing functions $q: \mathbb{R}_{>0} \rightarrow \mathbb{R}_{>0}$, which satisfy $q(t)>0$ for all $t>0$. A function $\gamma: \mathbb{R}_{\geq 0} \rightarrow \mathbb{R}_{\geq 0}$ is of class $\mathcal{G}$ if it is continuous, nondecreasing and zero at zero. It is of class $\mathcal{K}$ if it is of class $\mathcal{G}$ and strictly increasing; and it is of class $\mathcal{K}_{\infty}$ if it is of class $\mathcal{K}$ and unbounded. Functions of class $\mathcal{K}_{\infty}$ are invertible. Given two functions $\alpha(\cdot)$ and $\gamma(\cdot)$, we denote their composition and multiplication respectively as $\alpha \circ \gamma(\cdot)$ and $\alpha(\cdot) \cdot \gamma(\cdot)$. $|x|$ denotes the 1 -norm of a vector $x \in \mathbb{R}^{n}$, that is $|x|:=$ $\sum_{i=1}^{n}\left|x_{i}\right|$.

Motivated by the discussion on the previous section, we consider a parameterized family of discrete-time nonlinear systems of the following form:

$$
\begin{aligned}
x(k+1) & =F_{T}(x(k), u(k)) \\
y(k) & =h(x(k))
\end{aligned}
$$

where $x \in \mathbb{R}^{n}, u \in \mathbb{R}^{m}, y \in \mathbb{R}^{l}$ are respectively the state, input and output of the system. It is assumed that $F_{T}$ is well defined for all $x, u$ and sufficiently small $T$, $F_{T}(0,0)=0$ for all $T$ for which $F_{T}$ is defined, $h(0)=0$ and $F_{T}$ and $h$ are continuous. $T>0$ is the sampling period, which parameterizes the system and can be arbitrarily assigned. Non-parameterized discrete-time systems are a special case of (9) when $T$ is constant (for instance $T=1$ ). The following definition is a very compact way of defining various different properties to which our results apply.

Definition 3.1 The system (9) is $\left(V_{T}, \underline{\alpha}, \bar{\alpha}, \alpha, \lambda, \sigma\right)$ semiglobally practically input-output to state stable $\left(\left(V_{T}, \underline{\alpha}, \bar{\alpha}, \alpha, \lambda, \sigma\right)-S P-I O S S\right) \quad$ with measuring functions, if there exist functions $\underline{\alpha}, \bar{\alpha}, \alpha \in \mathcal{K}_{\infty}$, and $\lambda$, $\sigma \in \mathcal{G}$, functions $w_{\underline{\alpha}}: \mathbb{R}^{n} \rightarrow \mathbb{R}^{n_{\underline{\alpha}}}, w_{\bar{\alpha}}: \mathbb{R}^{n} \rightarrow \mathbb{R}^{n_{\bar{\alpha}}}$, $w_{\alpha}: \mathbb{R}^{n} \rightarrow \mathbb{R}^{n_{\alpha}}, w_{\lambda}: \mathbb{R}^{n} \rightarrow \mathbb{R}^{n_{\lambda}}, w_{\sigma}: \mathbb{R}^{m} \rightarrow \mathbb{R}^{n_{\sigma}}$, $w_{x}: \mathbb{R}^{n} \rightarrow \mathbb{R}^{n_{x}}, w_{u}: \mathbb{R}^{m} \rightarrow \mathbb{R}^{n_{u}}$, and for any triple of strictly positive real numbers $\Delta_{x}, \Delta_{u}, \nu$, there exists $T^{*}>0$ and for all $T \in\left(0, T^{*}\right)$ there exists a smooth function $V_{T}: \mathbb{R}^{n} \rightarrow \mathbb{R}_{>0}$ such that for all $\left|w_{x}(x)\right| \leq \Delta_{x}$, $\left|w_{u}(u)\right| \leq \Delta_{u}$ the following holds:

$$
\begin{aligned}
\underline{\alpha}\left(\left|w_{\underline{\alpha}}(x)\right|\right) & \leq V_{T}(x) \leq \bar{\alpha}\left(\left|w_{\bar{\alpha}}(x)\right|\right) \\
V_{T}\left(F_{T}(x, u)\right)-V_{T}(x) & \leq-T \alpha\left(\left|w_{\alpha}(x)\right|\right) \\
+T \lambda\left(\left|w_{\lambda}(x)\right|\right) & +T \sigma\left(\left|w_{\sigma}(u)\right|\right)+T \nu .
\end{aligned}
$$

The functions $w_{\alpha}, w_{\bar{\alpha}}, w_{\alpha}, w_{\lambda}, w_{\sigma}, w_{x}$ and $w_{u}$ are called measuring functions; $\underline{\alpha}, \bar{\alpha}, \alpha, \lambda, \sigma$ are called bounding functions; $\alpha, \lambda, \sigma$ are called supply functions; and $V_{T}$ is called a SP-IOSS Lyapunov function. If $T^{*}>0$ exists such that (10) and (11), with $\nu=0$, hold for all $T \in$ $\left(0, T^{*}\right), x \in \mathbb{R}^{n}, u \in \mathbb{R}^{m}$, the property holds globally and the system (9) is $\left(V_{T}, \underline{\alpha}, \bar{\alpha}, \alpha, \lambda, \sigma\right)$-IOSS with measuring functions.

Often, when all functions are clear from the context, we refer to the property defined in Definition 3.1 as SPIOSS (or IOSS if the property holds globally). Moreover, if the system is SP-IOSS (respectively IOSS) with 
$\lambda=0$ then we say that the system is SP-ISS (respectively ISS). SP-IOSS with measuring functions is quite a general notion that covers a range of different properties of nonlinear discrete-time systems, such as stability, input to state stability, detectability, output to state stability, etc. For example, by letting $\lambda=0, \sigma=0$ and $w_{\underline{\alpha}}(x)=w_{\bar{\alpha}}(x)=w_{\alpha}(x)=x$, we obtain the standard Lyapunov characterization for asymptotic stability of (9). By letting $\lambda=0, w_{\alpha}(x)=w_{\bar{\alpha}}(x)=w_{\alpha}(x)=x$, and $w_{\sigma}(u)=u$, we obtain a Lyapunov characterization for (semiglobal practical) ISS. The reason for introducing such a general property in Definition 3.1 is that we will apply our results to a range of its different special cases (see Section 4) for particular choices of $\lambda, \sigma$ and the measuring functions.

When using the SP-IOSS property of Definition 3.1 to check if a certain property (such as stability, input to state stability or some other special cases of SP-IOSS property) holds, one usually needs to have that all bounding functions and the corresponding measuring functions satisfy appropriate conditions. For example, if we want to check global asymptotic stability of the origin of the input-free system (9) then we need to have:

$$
\begin{aligned}
\underline{\alpha}\left(\left|w_{\underline{\alpha}}(x)\right|\right) & \leq V_{1 T}(x) \leq \bar{\alpha}\left(\left|w_{\bar{\alpha}}(x)\right|\right) \\
V_{1 T}\left(F_{T}(x, 0)\right)-V_{1 T}(x) & \leq-T \alpha\left(\left|w_{\alpha}(x)\right|\right),
\end{aligned}
$$

for all $x \in \mathbb{R}^{n}$ and $T \in\left(0, T^{*}\right)$, for some $T^{*}>0 ; \underline{\alpha}, \bar{\alpha} \in$ $\mathcal{K}_{\infty}$ and $\alpha$ is positive definite; $\left|w_{\underline{\alpha}}(x)\right|$ is positive definite and radially unbounded; and $\left|w_{\alpha}(x)\right|$ is positive definite.

\section{Main results}

In this section, we state our main results, which consist of two main theorems (Theorems 4.1 and 4.2), where we show two partial constructions of a SP-IOSS Lyapunov function from two auxiliary Lyapunov functions. Several special cases following from our main results are presented as corollaries. We first present Lemma 4.1, which is instrumental in proving our main results. The lemma is a discrete-time version, as well as a generalization, of the lemma on changing supply rates for IOSS continuous-time systems in [1]. Lemma 4.1 also generalizes the result of [26] on changing supply rates for ISS discrete-time systems. We use the following construction that has also been used in $[1,31]$. Given an arbitrary $q \in \mathcal{S N}$, we define:

$$
\rho(s):=\int_{0}^{s} q(\tau) d \tau,
$$

where it is easy to see that $\rho \in \mathcal{K}_{\infty}$ and $\rho$ is smooth. Suppose that we have a SP-IOSS Lyapunov function $V_{T}$ for a system, and consider a new function $\rho\left(V_{T}\right)$. Lemma 4.1 states the conditions under which the new function is also a SP-IOSS Lyapunov function for the system.
Lemma 4.1 Let the following conditions be satisfied: 1. System (9) is $\left(V_{T}, \underline{\alpha}, \bar{\alpha}, \alpha, \lambda, \sigma\right)$-SP-IOSS with measuring functions $w_{\underline{\alpha}}, w_{\bar{\alpha}}, w_{\alpha}, w_{\lambda}, w_{\sigma}, w_{x}$ and $w_{u}$.

2. There exist $\underline{\kappa}, \bar{\kappa} \in \mathcal{K}_{\infty}$ such that $\underline{\kappa}\left(\left|w_{\alpha}(x)\right|\right) \leq\left|w_{\underline{\alpha}}(x)\right|$ and $\left|w_{\bar{\alpha}}(x)\right| \leq \bar{\kappa}\left(\left|w_{\alpha}(x)\right|\right), \forall x \in \mathbb{R}^{n}$.

3. For any strictly positive real numbers $\Delta_{x}, \Delta_{u}$ there exist strictly positive real numbers $M$ and $T^{*}$ such that

$$
\begin{aligned}
& \left|w_{x}(x)\right| \leq \Delta_{x},\left|w_{u}(u)\right| \leq \Delta_{u}, T \in\left(0, T^{*}\right) \Longrightarrow \\
& \max \left\{\left|w_{\bar{\alpha}}\left(F_{T}(x, u)\right)\right|,\left|w_{\bar{\alpha}}(x)\right|,\left|w_{\lambda}(x)\right|,\left|w_{\sigma}(u)\right|\right\} \leq M
\end{aligned}
$$

Then for any $q \in \mathcal{S N}$ and $\rho \in \mathcal{K}_{\infty}$ defined by (13) there exist $\underline{\alpha}^{\prime}, \bar{\alpha}^{\prime}, \alpha^{\prime}, \lambda^{\prime}, \sigma^{\prime}$ such that the system (9) is $\left(\rho\left(V_{T}\right), \underline{\alpha}^{\prime}, \bar{\alpha}^{\prime}, \alpha^{\prime}, \lambda^{\prime}, \sigma^{\prime}\right)$-SP-IOSS with the same measuring functions, where $\underline{\alpha}^{\prime}(s)=\rho \circ \underline{\alpha}(s), \bar{\alpha}^{\prime}(s)=\rho \circ \bar{\alpha}(s)$, $\alpha^{\prime}(s)=\frac{1}{4} q \circ \frac{1}{2} \underline{\alpha} \circ \underline{\kappa}(s) \cdot \alpha(s), \lambda^{\prime}(s)=2 q \circ \theta_{\lambda}(s) \cdot \lambda(s)$, $\sigma^{\prime}(s)=2 q \circ \theta_{\sigma}(s) \cdot \sigma(s), \theta_{\sigma}(s):=\bar{\alpha} \circ \bar{\kappa} \circ \alpha^{-1} \circ 4 \sigma(s)+2 \sigma(s)$ and $\theta_{\lambda}(s):=\bar{\alpha} \circ \bar{\kappa} \circ \alpha^{-1} \circ 4 \lambda(s)+2 \lambda(s)$.

Lemma 4.1 provides us with some flexibility when constructing a SP-IOSS Lyapunov function $V_{T}$ from two Lyapunov functions as what we will do in Theorems 4.1 and 4.2. We prove the result for semiglobal practical IOSS since this is a property that naturally arises when an approximate discrete-time model is used for controller design of a sampled-data nonlinear systems (see Example 6 in the next section). Some of the conditions of Lemma 4.1 are rather technical but they were considered in order to prove the result in a considerable generality that allows us to unify presentation of several different results.

Remark 4.1 It is instructive to discuss the third condition of Lemma 4.1 since it appears to be the least intuitive. Let us first consider stability of the origin of the inputfree system (9). In this case, the conditions (12) need to hold and we can assume without loss of generality that $w_{\underline{\alpha}}(x)=w_{\bar{\alpha}}(x)=w_{\alpha}(x)=w_{x}(x)=x$. In this case the third condition of Lemma 4.1 holds if $F_{T}(x, 0)$ is bounded on compact sets, uniformly in $T \in\left(0, T^{*}\right)$. This holds if $F_{T}(0,0)=0$ for all $T \in\left(0, T^{*}\right)$ and $F_{T}(x, 0)$ is continuous in $x$, uniformly in $T \in\left(0, T^{*}\right)$. This condition is rather natural to use and it is often assumed in the literature (see for instance [12]). Suppose now that (12) hold with $w_{\underline{\alpha}}(x)=w_{\bar{\alpha}}(x)=w_{\alpha}(x)=w_{x}(x)=|x|_{\mathcal{A}}$, where $\mathcal{A}$ is a non-empty closed set. In this case, the condition 3 of Lemma 4.1 requires that for any $\Delta_{x}$ there exists $M$ and $T^{*}$ such that

$$
|x|_{\mathcal{A}} \leq \Delta_{x}, T \in\left(0, T^{*}\right) \Longrightarrow\left|F_{T}(x, 0)\right|_{\mathcal{A}} \leq M .
$$

This condition also appears to be natural and similar conditions have been used in the literature [35].

We can also state a similar result to Lemma 4.1, when the IOSS property holds globally, that is when the system $(9)$ is $\left(V_{T}, \underline{\alpha}, \bar{\alpha}, \alpha, \lambda, \sigma\right)$-IOSS with measuring functions. It is interesting that in this case the third condition of Lemma 4.1 is not needed to prove the result. 
Corollary 4.1 Let the following conditions be satisfied: 1. System (9) is $\left(V_{T}, \underline{\alpha}, \bar{\alpha}, \alpha, \lambda, \sigma\right)$-IOSS with measuring functions $w_{\underline{\alpha}}, w_{\bar{\alpha}}, w_{\alpha}, w_{\lambda}$ and $w_{\sigma}$.

2. There exist $\underline{\kappa}, \bar{\kappa} \in \mathcal{K}_{\infty}$ such that $\underline{\kappa}\left(\left|w_{\alpha}(x)\right|\right) \leq\left|w_{\underline{\alpha}}(x)\right|$ and $\left|w_{\bar{\alpha}}(x)\right| \leq \bar{\kappa}\left(\left|w_{\alpha}(x)\right|\right), \forall x \in \mathbb{R}^{n}$.

Then for any $q \in \mathcal{S} \mathcal{N}$ and $\rho \in \mathcal{K}_{\infty}$ defined by (13) there exist $\alpha^{\prime}, \bar{\alpha}^{\prime}, \alpha^{\prime}, \lambda^{\prime}, \sigma^{\prime}$ such that the system (9) is $\left(\rho\left(V_{T}\right), \underline{\alpha}^{\prime}, \bar{\alpha}^{\prime}, \alpha^{\prime}, \lambda^{\prime}, \sigma^{\prime}\right)$-IOSS with the same measuring functions, where $\underline{\alpha}^{\prime}, \bar{\alpha}^{\prime}, \alpha^{\prime}, \lambda^{\prime}, \sigma^{\prime}$ are the same as in Lemma 4.1.

We present our main results below. Note that Theorem 4.1 is a discrete-time version, as well as generalization, of the continuous-time results in [1], whereas Theorem 4.2 has appeared in a simpler form in [26], which is a discretetime version of [31], when $\lambda=0, w_{\underline{\alpha}}(x)=w_{\bar{\alpha}}(x)=$ $w_{\alpha}(x)=x, w_{\sigma}(u)=u$ and all properties hold globally.

Theorem 4.1 Suppose that:

1. system (9) is $\left(V_{1 T}, \underline{\alpha}_{1}, \bar{\alpha}_{1}, \alpha_{1}, \sigma_{1}\right)$-SP-ISS with measuring functions $w_{\underline{\alpha}_{1}}, w_{\bar{\alpha}_{1}}, w_{\alpha_{1}}, w_{\sigma_{1}}, w_{x_{1}}, w_{u_{1}}$;

2. system (9) is $\left(\bar{V}_{2 T}, \underline{\alpha}_{2}, \bar{\alpha}_{2}, \alpha_{2}, \lambda_{2}, \sigma_{2}\right)$-SP-IOSS with measuring functions $w_{\underline{\alpha}_{2}}, w_{\bar{\alpha}_{2}}, w_{\alpha_{2}}, w_{\lambda_{2}}, w_{\sigma_{2}}, w_{x_{2}}, w_{u_{2}}$, and there exist $\underline{\kappa}_{2}, \bar{\kappa}_{2} \stackrel{\underline{\alpha}_{2}}{\in} \mathcal{K}_{\infty}$, such that the second and third conditions of Lemma 4.1 hold;

3. there exist $\gamma_{1}, \gamma_{2}, \gamma_{3} \in \mathcal{K}_{\infty}$ such that $\left|w_{\lambda_{2}}(x)\right| \leq$ $\gamma_{1}\left(\left|w_{\alpha_{1}}(x)\right|\right), \quad\left|w_{x_{2}}(x)\right| \leq \gamma_{2}\left(\left|w_{x_{1}}(x)\right|\right), \quad\left|w_{u_{2}}(u)\right| \leq$ $\gamma_{3}\left(\left|w_{u_{1}}(u)\right|\right)$ for all $x \in \mathbb{R}^{n}, u \in \mathbb{R}^{m}$;

4. $\lim \sup _{s \rightarrow+\infty} \frac{\lambda_{2}(s)}{\alpha_{1}(s)}<+\infty$.

Then there exists $\rho \in \mathcal{K}_{\infty}$ such that the system (9) is $\left(V_{T}, \underline{\alpha}, \bar{\alpha}, \alpha, \sigma\right)-S P-I S S$ with new measuring functions $w_{\underline{\alpha}}, w_{\bar{\alpha}}, w_{\alpha}, w_{\sigma}, w_{x}, w_{u}$, where

$$
V_{T}=V_{1 T}+\rho\left(V_{2 T}\right),
$$

and the new measuring functions are

$$
\begin{aligned}
w_{\underline{\alpha}}(x) & :=\left|w_{\underline{\alpha}_{1}}(x)\right|+\left|w_{\alpha_{2}}(x)\right|, w_{\alpha}(x):=\left|w_{\alpha_{2}}(x)\right|, \\
w_{\bar{\alpha}}(x) & :=\left|w_{\bar{\alpha}_{1}}(x)\right|+\left|w_{\bar{\alpha}_{2}}(x)\right|, w_{x}(x):=w_{x_{1}}(x), \\
w_{\sigma}(u) & :=\left|w_{\sigma_{1}}(u)\right|+\left|w_{\sigma_{2}}(u)\right|, w_{u}(u):=w_{u_{1}}(u) .
\end{aligned}
$$

Remark 4.2 In order to carry out the construction given in Theorem 4.1, the measuring functions for $V_{1 T}$ and $V_{2 T}$ have to satisfy condition 3 of the theorem. Indeed, some measuring functions of $V_{1 T}$ have to "match" certain measuring functions of $V_{2 T}$. To better understand these conditions, we consider a system with the output $y=h(x)$. For simplicity, let $\lambda_{1}=\sigma_{1}=\sigma_{2} \equiv 0$. Suppose that $w_{\underline{\alpha}_{1}}(x)=w_{\bar{\alpha}_{1}}(x)=w_{\underline{\alpha}_{2}}(x)=w_{\bar{\alpha}_{2}}(x)=w_{\alpha_{2}}(x)=x$ and $w_{\lambda_{2}}(x)=w_{\alpha_{1}}(x)=y$. In this case, condition 3 holds. This is a familiar situation where the first difference of $V_{1 T}$ is negative semidefinite, that is $\Delta V_{1 T} \leq-T \alpha_{1}(|y|)$. Moreover, $V_{2 T}$ satisfies $\Delta V_{2 T} \leq-T \alpha_{2}(|x|)+T \lambda_{2}(|y|)$, which is a particular detectability property of the system w.r.t the output $y$. More examples and important special cases are presented in Section 5.
In the next result, we consider a stronger condition for the Lyapunov function $V_{1 T}$, so that we can relax the condition 4 of Theorem 4.1.

Theorem 4.2 Suppose that:

1. system (9) is $\left(V_{1 T}, \underline{\alpha}_{1}, \bar{\alpha}_{1}, \alpha_{1}, \sigma_{1}\right)$-SP-ISS with measuring functions $w_{\underline{\alpha}_{1}}, w_{\bar{\alpha}_{1}}, w_{\alpha_{1}}, w_{\sigma_{1}}, w_{x_{1}}, w_{u_{1}}$ and there exist $\underline{\kappa}_{1}, \bar{\kappa}_{1} \in \mathcal{K}_{\infty}$, such that the second and third conditions of Lemma 4.1 hold;

2. system (9) is $\left(V_{2 T}, \underline{\alpha}_{2}, \bar{\alpha}_{2}, \alpha_{2}, \lambda_{2}, \sigma_{2}\right)$-SP-IOSS with measuring functions $w_{\underline{\alpha}_{2}}, w_{\bar{\alpha}_{2}}, w_{\alpha_{2}}, w_{\lambda_{2}}, w_{\sigma_{2}}, w_{x_{2}}, w_{u_{2}}$ and there exist $\underline{\kappa}_{2}, \bar{\kappa}_{2} \in \overline{\mathcal{K}}_{\infty}$, such that the second and third condition of Lemma 4.1 hold;

3. the item 3 of Theorem 4.1 holds;

Then there exist $\rho_{1}, \rho_{2} \in \mathcal{K}_{\infty}$ such that the system (9) is $\left(V_{T}, \underline{\alpha}, \bar{\alpha}, \alpha, \sigma\right)-S P$-ISS with new measuring functions $w_{\underline{\alpha}}, w_{\bar{\alpha}}, w_{\alpha}, w_{\sigma}, w_{x}, w_{u}$, where

$$
V_{T}=\rho_{1}\left(V_{1 T}\right)+\rho_{2}\left(V_{2 T}\right)
$$

and the new measuring functions are $w_{\underline{\alpha}}, w_{\bar{\alpha}}, w_{x}, w_{\sigma}$ and $w_{u}$ are given in (16) and $w_{\alpha}(x):=\left|w_{\alpha_{1}}(x)\right|+\left|w_{\alpha_{2}}(x)\right|$

Remark 4.3 We note that in Theorems 4.1 and 4.2 we concentrate only on verifying conditions similar to (6), (7). However, we note that if the functions $V_{1 T}$ and $V_{2 T}$ satisfy the local Lipschitz condition (8), then the new Lyapunov function constructed using either (15) or (17) would also satisfy the local Lipschitz condition. Hence, results of Theorem 4.1 and 4.2 can be used to verify the first condition of Theorem 2.1. Additionally, since we assume that $q_{1}(\cdot)$ and $q_{2}(\cdot)$ are smooth, then if $V_{1 T}$ and $V_{2 T}$ are smooth functions, then so is $V_{T}$. Having smooth $V_{T}$ is important in some cases, such as in the design using backstepping [25].

Note that the main difference between Theorems 4.1 and 4.2 is that in Theorem 4.1 we cannot apply Lemma 4.1 to the Lyapunov function $V_{1 T}$, since the second and third conditions of the lemma do not hold. Consequently, we need an extra condition on the bounding functions (condition 4 in Theorem 4.1) and we use a less general construction (15) than in Theorem 4.2 where we use (17). As a consequence of Corollary 4.1, we can also state global versions of Theorems 4.1 and 4.2, if both $V_{1 T}$ and $V_{2 T}$ characterize IOSS properties of the system (9) in a global sense.

Corollary 4.2 Suppose that all conditions of Theorem 4.1 hold globally. Then, there exists $\rho \in \mathcal{K}_{\infty}$ such that the system (9) is $\left(V_{T}, \underline{\alpha}, \bar{\alpha}, \alpha, \sigma\right)$-ISS where $V_{T}$ is given by (15) and the new measuring functions $w_{\underline{\alpha}}, w_{\bar{\alpha}}, w_{\alpha}$, $w_{\sigma}, w_{x}, w_{u}$ are given in Theorem 4.1.

Corollary 4.3 Suppose that all conditions of Theorem 4.2 hold globally. Then, there exist $\rho_{1}, \rho_{2} \in \mathcal{K}_{\infty}$ such that the system (9) is $\left(V_{T}, \underline{\alpha}, \bar{\alpha}, \alpha, \sigma\right)$-ISS where $V_{T}$ is given by (17) and the new measuring functions $w_{\underline{\alpha}}, w_{\bar{\alpha}}, w_{\alpha}$, $w_{\sigma}, w_{x}, w_{u}$ are given in Theorem 4.2. 


\section{Applications}

In this section we show how our results can be specialized to deal with several important situations. We also emphasize that our results are quite general and they have potential for other applications. We only include the proof of Corollary 5.1, since the proofs of other Corollaries in this section follow similar steps.

\subsection{A LaSalle criterion for SP-ISS}

In this subsection, we present a novel result which is a discrete-time version of the continuous-time result presented in [1]. This result is a direct consequence of Theorem 4.1. We use this result for the case study in Section 6 to design a digital controller for a two link manipulator via its Euler approximate model.

We recall the quasi input to state stability (qISS) property and input output to state stability (IOSS) property from [1], and recall the condition

$$
\lim \sup _{s \rightarrow+\infty} \frac{\lambda_{2}(s)}{\alpha_{1}(s)}<+\infty
$$

that has been used in the result of [1]. Using Theorem 4.1 we can state a semiglobal practical version of this result for parameterized discrete-time systems (9). In particular, we show that semiglobal practical qISS, semiglobal practical IOSS and the condition (18) imply semiglobal practical ISS. We use the following assumption:

Assumption 5.1 For any strictly positive real numbers $\Delta_{x}, \Delta_{u}$ there exist strictly positive real numbers $M$ and $T^{*}$ such that $|x| \leq \Delta_{x},|u| \leq \Delta_{u}, T \in\left(0, T^{*}\right)$ implies $\left|F_{T}(x, u)\right| \leq M$

We state now a discrete-time version of the result in [1].

Corollary 5.1 Consider the system (9). Suppose that Assumption 5.1 holds, and there exist $\underline{\alpha}_{1}, \bar{\alpha}_{1}, \alpha_{1}, \underline{\alpha}_{2}, \bar{\alpha}_{2}, \alpha_{2} \in$ $\mathcal{K}_{\infty}$, and $\sigma_{1}, \lambda_{2}, \sigma_{2} \in \mathcal{G}$ such that:

1. for any triple of strictly positive real numbers $\left(\Delta_{x}, \Delta_{u}, \nu\right)$ there exists $T^{*}>0$ and for any $T \in\left(0, T^{*}\right)$ there exist $V_{1 T}: \mathbb{R}^{n} \rightarrow \mathbb{R}_{>0}$ and $V_{2 T}: \mathbb{R}^{n} \rightarrow \mathbb{R}_{>0}$ such that for all $|x| \leq \Delta_{x},|u| \leq \Delta_{u}, T \in\left(0, T^{*}\right)$ we have the following:

$S P-q I S S$ :

$$
\begin{aligned}
& \underline{\alpha}_{1 T}\left(F_{T}\right)-V_{1 T}(x) \leq T\left(-\alpha_{1}(|y|)+\sigma_{1}(|u|)+\nu\right) .
\end{aligned}
$$

SP-IOSS: $\quad \underline{\alpha}_{2}(|x|) \leq V_{2 T}(x) \leq \bar{\alpha}_{2}(|x|)$

$V_{2 T}\left(F_{T}\right)-V_{2 T}(\bar{x}) \leq T\left(-\alpha_{2}(|x|)+\lambda_{2}(|y|)+\sigma_{2}(|u|)+\nu\right)$. 2. the condition (18) holds.

Then, there exist $\underline{\alpha}, \bar{\alpha}, \alpha \in \mathcal{K}_{\infty}$ and $\sigma \in \mathcal{G}$ such that for any triple of strictly positive real numbers $\left(\widetilde{\Delta}_{x}, \widetilde{\Delta}_{u}, \widetilde{\nu}\right)$ there exists $\widetilde{T}>0$ and for any $T \in(0, \widetilde{T})$ there exist $V_{T}: \mathbb{R}^{n} \rightarrow \mathbb{R}_{\geq 0}$ such that for all $|x| \leq \widetilde{\Delta}_{x},|u| \leq \widetilde{\Delta}_{u}$, $T \in(0, \widetilde{T})$ we have:

SP-ISS: $\quad \underline{\alpha}(|x|) \leq V_{T}(x) \leq \bar{\alpha}(|x|)$

$V_{T}\left(F_{T}\right)-V_{T}(x) \leq T(-\alpha(|x|)+\sigma(|u|)+\widetilde{\nu})$.
Proof of Corollary 5.1: It can be seen immediately that all conditions of Theorem 4.1 hold, by noting that: (i) the system $(9)$ is $\left(V_{1 T}, \underline{\alpha}_{1}, \bar{\alpha}_{1}, \alpha_{1}, \sigma_{1}\right)$-SP-ISS with measuring functions $w_{\underline{\alpha}_{1}}(x)=w_{\bar{\alpha}_{1}}(x)=w_{x_{1}}(x)=x$, $w_{\alpha_{1}}(x)=h(x)=y, w_{\sigma_{1}}(u)=w_{u_{1}}(u)=u$; (ii) the system (9) is $\left(V_{2 T}, \underline{\alpha}_{2}, \bar{\alpha}_{2}, \alpha_{2}, \lambda_{2}, \sigma_{2}\right)$-SP-IOSS with measuring functions $w_{\underline{\alpha}_{2}}(x)=w_{\bar{\alpha}_{2}}(x)=w_{\alpha_{2}}(x)=$ $w_{x_{2}}(x)=x, w_{\lambda_{2}}(x)=h(x)=y$ and $w_{\sigma_{2}}(u)=w_{u_{2}}(u)=$ $u$; the second condition of Lemma 4.1 holds since $w_{\underline{\alpha}_{2}}(x)=w_{\bar{\alpha}_{2}}(x)=w_{\alpha_{2}}(x)$; from Assumption 5.1 and Remark 4.1 we have that the third condition of Lemma 4.1 holds; hence, the second condition of Theorem 4.1 holds; (iii) the third condition of Theorem 4.1 holds since $w_{\alpha_{1}}(x)=w_{\lambda_{2}}(x)=h(x)=y, w_{x_{1}}(x)=w_{x_{2}}(x)=x$ and $w_{u_{1}}(u)=w_{u_{2}}(u)=u$ for all $x \in \mathbb{R}^{n}, u \in \mathbb{R}^{m}$; (iv) the fourth condition of Theorem 4.1 follows trivially from the second condition of the corollary. Therefore, applying Theorem 4.1 and defining the new SPISS Lyapunov function $V_{T}$ as in (15), we obtain that the system (9) is SP-ISS with measuring functions $w_{\underline{\alpha}}(x)=w_{\bar{\alpha}}(x)=w_{\alpha}(x)=|x|, w_{x}(x)=x, w_{\sigma}(u)=|u|$, and $w_{u}(u)=u$. It is obvious that $\gamma_{2}=\gamma_{3}=I d$. Since $h$ is continuous and $h(0)=0$, there exists $\gamma_{1} \in \mathcal{K}_{\infty}$ such that $|y| \leq \gamma_{1}(|x|)$, and this completes the proof.

\subsection{SP-ISS of time-varying cascade-connected systems}

A novel result on SP-ISS for time-varying discrete-time cascade-connected system is presented in this subsection. This result is a direct consequence of Theorem 4.2 and it generalizes the main result of [26] in two directions: (i) the result is stated for semiglobal practical ISS (only global stability was considered in [26]); (ii) the result is stated for time-varying cascade-connected systems (only time-invariant cascade-connected systems were considered in [26]). We note that similar non Lyapunov based proof of the same result can be found in [12] for non parameterized discrete-time systems.

Consider the time-varying discrete-time system:

$$
\begin{aligned}
& x(k+1)=F_{T}(k, x(k), z(k), u(k)) \\
& z(k+1)=G_{T}(k, z(k), u(k)),
\end{aligned}
$$

where $x \in \mathbb{R}^{n_{x}}, z \in \mathbb{R}^{n_{z}}$ and $u \in \mathbb{R}^{m}$. The state of the overall system is denoted as $\tilde{x}:=\left(x^{T} z^{T}\right)^{T}, \tilde{x} \in \mathbb{R}^{n}$, where $n:=n_{x}+n_{z}$. We will assume the following:

Assumption 5.2 For any strictly positive real numbers $\Delta_{\tilde{x}}, \Delta_{u}$ there exist strictly positive real numbers $M$ and $T^{*}$ such that

$$
\begin{aligned}
|\tilde{x}| \leq & \Delta_{\tilde{x}},|u| \leq \Delta_{u}, T \in\left(0, T^{*}\right), k \geq 0 \\
& \Longrightarrow \max \left\{\left|F_{T}(k, x, z, u)\right|,\left|G_{T}(k, z, u)\right|\right\} \leq M .
\end{aligned}
$$

The family of systems (19) is not in the form (9) which is time invariant. However, we can still apply results of our paper in the following way. We introduce an augmented 
time-invariant system in the following way:

$$
\begin{aligned}
& x(k+1)=F_{T}(p(k), x(k), z(k), u(k)) \\
& z(k+1)=G_{T}(p(k), z(k), u(k)) \\
& p(k+1)=p(k)+1
\end{aligned}
$$

where $p \in \mathbb{R}$ is a new state variable. Then it is standard to show that SP-ISS uniform of the time-varying system $(19)$ w.r.t. the origin $(x, z)=(0,0)$ can be deduced from semiglobal practical ISS of the time-invariant system (20) w.r.t. a non-compact set $\mathcal{A}:=\{(\tilde{x}, p): \tilde{x}=0\}$. Note also that we can write $|\tilde{x}|=|(\tilde{x}, p)|_{\mathcal{A}}$.

In the next result we show that SP-ISS Lyapunov function for the overall system (20) can be constructed from Lyapunov functions for individual subsystems in (20). In particular, we can state the following:

Corollary 5.2 Consider the system (19). Suppose that Assumption 5.2 holds and there exist $\underline{\alpha}_{1}, \bar{\alpha}_{1}, \alpha_{1}$, $\underline{\alpha}_{2}, \bar{\alpha}_{2}, \alpha_{2} \in \mathcal{K}_{\infty}$, and $\sigma_{1}, \lambda_{1}, \sigma_{2} \in \mathcal{G}$ such that for any triple of strictly positive real numbers $\left(\Delta_{\tilde{x}}, \Delta_{u}, \nu\right)$ there exists $T^{*}>0$ and for any $T \in\left(0, T^{*}\right)$ there exist $V_{1 T}: \mathbb{R} \times \mathbb{R}^{n_{x}} \rightarrow \mathbb{R}_{>0}$ and $V_{2 T}: \mathbb{R} \times \mathbb{R}^{n_{z}} \rightarrow \mathbb{R}_{>0}$ such that for all $|\tilde{x}| \leq \Delta_{\tilde{x}},|u| \leq \Delta_{u}, p \geq 0, T \in\left(0, T^{*}\right)$ we have the following:

$$
\begin{aligned}
\underline{\alpha}_{1}(|x|) & \leq V_{1 T}(p, x) \leq \bar{\alpha}_{1}(|x|) \\
V_{1 T}\left(p+1, F_{T}\right) & -V_{1 T}(p, x) \\
& \leq T\left(-\alpha_{1}(|x|)+\lambda_{1}(|z|)+\sigma_{1}(|u|)+\nu\right) \\
\underline{\alpha}_{2}(|z|) & \leq V_{2 T}(p, z) \leq \bar{\alpha}_{2}(|z|) \\
V_{2 T}\left(p+1, G_{T}\right)- & V_{2 T}(p, z) \leq T\left(-\alpha_{2}(|z|)+\sigma_{2}(|u|)+\nu\right) .
\end{aligned}
$$

Then, there exist $\underline{\alpha}, \bar{\alpha}, \alpha \in \mathcal{K}_{\infty}$ and $\sigma \in \mathcal{G}$ such that for any triple of strictly positive real numbers $\left(\widetilde{\Delta}_{\tilde{x}}, \widetilde{\Delta}_{u}, \widetilde{\nu}\right)$ there exists $\widetilde{T}>0$ and for any $T \in(0, \widetilde{T})$ there exist $V_{T}: \mathbb{R} \times \mathbb{R}^{n} \rightarrow \mathbb{R}_{\geq 0}$ such that for all $|\tilde{x}| \leq \widetilde{\Delta}_{\tilde{x}},|u| \leq \widetilde{\Delta}_{u}$, $p \geq 0, T \in(0, \tilde{T})$ we have:

$$
\begin{aligned}
& S \bar{P}-I S S: \quad \underline{\alpha}(|\tilde{x}|) \leq V_{T}(p, x, z) \leq \bar{\alpha}(|\tilde{x}|) \\
& V_{T}\left(p+1, F_{T}, G_{T}\right)-V_{T}(p, x, z) \\
& \leq T(-\alpha(|\tilde{x}|)+\sigma(|u|)+\widetilde{\nu}) .
\end{aligned}
$$

\subsection{SP-ISS via positive semidefinite Lyapunov func-} tions

The problem of checking stability using positive semidefinite Lyapunov functions has been considered in [5] for continuous-time systems and in [9] for discrete-time systems. The idea is to use a Lyapunov function $V(x)$, which is positive semidefinite, to check stability of a system. An approach taken in $[5,9]$ was to use a trajectorybased technique to prove stability of the origin of the system. In particular, besides appropriate conditions on the Lyapunov function, it was required in $[5,9]$ that all trajectories in the maximal invariant subset of the set $Z:=\{x: V(x)=0\}$ satisfy the $\epsilon-\delta$ definition of asymptotic stability (this property was referred to as conditional stability to the set $Z$ ).
We note that the results on stability of cascadeconnected systems in [26,31] and in the previous subsection can be interpreted as a special case of testing ISS using positive semidefinite Lyapunov functions. However, this approach is different from the one in [5,9] since an ISS Lyapunov function is constructed explicitly from ISS and IOSS Lyapunov functions of each subsystem. The advantage of the approach of $[26,31]$ is that it leads to a construction of a Lyapunov function for the overall system, whereas the disadvantage is that it requires usually stronger conditions and it appears to apply only to a special class of cascade-connected systems. However, we show here that the same approach can be used with few modifications to test semiglobal practical ISS of general parameterized discrete-time systems (9) that are not in the cascade form. In particular, we can state:

Corollary 5.3 Consider the family of systems (9). Suppose that Assumption 5.1 holds and there exist $\underline{\alpha}_{1}, \bar{\alpha}_{1}, \alpha_{1}$, $\underline{\alpha}_{2}, \bar{\alpha}_{2}, \alpha_{2} \in \mathcal{K}_{\infty}, \sigma_{1}, \lambda_{1}, \sigma_{2} \in \mathcal{G}$ and positive semidefinite functions $W_{1}: \mathbb{R}^{n} \rightarrow \mathbb{R}_{\geq 0}$ and $W_{2}: \mathbb{R}^{n} \rightarrow \mathbb{R}_{\geq 0}$, with $W_{1}(x)+W_{2}(x)$ is positive definite and radially unbounded, such that for any triple of strictly positive real numbers $\left(\Delta_{x}, \Delta_{u}, \nu\right)$ there exists $T^{*}>0$ and for any $T \in$ $\left(0, T^{*}\right)$ there exist $V_{1 T}: \mathbb{R}^{n} \rightarrow \mathbb{R}_{>0}$ and $V_{2 T}: \mathbb{R}^{n} \rightarrow \mathbb{R}_{>0}$ such that for all $|x| \leq \Delta_{x},|u| \leq \bar{\Delta}_{u}, T \in\left(0, T^{*}\right)$ we have the following:

$$
\begin{aligned}
\underline{\alpha}_{1}\left(W_{1}(x)\right) & \leq V_{1 T}(x) \leq \bar{\alpha}_{1}\left(W_{1}(x)\right) \\
V_{1 T}\left(F_{T}\right)-V_{1 T}(x) & \leq T\left(-\alpha_{1}\left(W_{1}(x)\right)\right. \\
& \left.+\lambda_{1}\left(W_{2}(x)\right)+\sigma_{1}(|u|)+\nu\right), \\
& \\
V_{2 T}\left(F_{T}\right)-V_{2 T}(x) \leq & T\left(-W_{2}\left(W_{2}(x)\right)+V_{2 T}(x) \leq \bar{\alpha}_{2}\left(W_{2}(x)\right)\right. \\
&
\end{aligned}
$$

Then, there exist $\underline{\alpha}, \bar{\alpha}, \alpha \in \mathcal{K}_{\infty}$ and $\sigma \in \mathcal{G}$ such that for any triple of strictly positive real numbers $\left(\widetilde{\Delta}_{x}, \widetilde{\Delta}_{u}, \widetilde{\nu}\right)$ there exists $\widetilde{T}>0$ and for any $T \in(0, \widetilde{T})$ there exist $V_{T}: \mathbb{R}^{n} \rightarrow \mathbb{R}_{\geq 0}$ such that for all $|x| \leq \widetilde{\Delta}_{x},|u| \leq \widetilde{\Delta}_{u}$, $T \in(0, \widetilde{T})$ we have:

$$
\begin{aligned}
& \text { SP-ISS: } \quad \underline{\alpha}(|x|) \leq V_{T}(x) \leq \bar{\alpha}(|x|) \\
& V_{T}\left(F_{T}\right)-\bar{V}_{T}(x) \leq T(-\alpha(|x|)+\sigma(|u|)+\widetilde{\nu}) .
\end{aligned}
$$

5.4 Observer-based input to state stabilization of discrete-time systems

Observer-based stabilization of discrete-time nonlinear systems that was considered in $[13,14]$ uses a very similar construction to the ones considered in this paper. It was shown in $[13,14]$ that if a discrete-time plant can be robustly stabilized with full state feedback (in an ISS sense) and there exists an observer for the system satisfying appropriate Lyapunov conditions (that is, the system is weakly detectable), then the plant is also stabilized using the controller/observer pair where the controller uses the state estimate obtained from the observer. Both local and global results were considered in $[13,14]$.

In this subsection, we show that our results, particularly Theorem 4.2, can be used to generalize results of 
$[13,14]$ in two directions: (i) we present results on observer based input to state stabilization of discrete-time systems (in $[13,14]$ only stabilization was considered); (ii) results on semiglobal practical ISS of parameterized systems (9) are presented (in [13,14] only global and local stabilization of non-parameterized discrete-time systems were considered).

We consider the parameterized family of plants:

$$
\begin{aligned}
x(k+1) & =F_{T}(x(k), u(k), v(k)) \\
y(k) & =h(x(k)),
\end{aligned}
$$

where $u$ and $v$ are respectively the control and exogenous inputs, with the following observer and controller respectively

$$
\begin{aligned}
z(k+1) & =G_{T}(z(k), h(x(k)), u(k), v(k)), \\
u(k) & =\phi_{T}(z(k))
\end{aligned}
$$

that are defined for sufficiently small $T$. Let $\tilde{x}:=$ $\left(x^{T} z^{T}\right)^{T}$, and we assume the following:

Assumption 5.3 For any strictly positive real numbers $\Delta_{\tilde{x}}, \Delta_{u}, \Delta_{v}$ there exist strictly positive real numbers $M$ and $T^{*}$ such that

$|\tilde{x}| \leq \Delta_{\tilde{x}},|u| \leq \Delta_{u},|v| \leq \Delta_{v}, T \in\left(0, T^{*}\right)$

$\Longrightarrow \max \left\{\left|F_{T}(x, u, v)\right|,\left|G_{T}(x, z, u, v)\right|,\left|\phi_{T}(z)\right|\right\} \leq M$.

Then, we can state the following result:

Corollary 5.4 Consider the family of systems (21), (22) and (23). Suppose that Assumption 5.3 holds and there exist $\underline{\alpha}_{1}, \bar{\alpha}_{1}, \alpha_{1}, \underline{\alpha}_{2}, \bar{\alpha}_{2}, \alpha_{2} \in \mathcal{K}_{\infty}, \sigma_{1}, \lambda_{1}, \sigma_{2} \in \mathcal{G}$, such that for any triple of strictly positive real numbers $\left(\Delta_{\tilde{x}}, \Delta_{v}, \nu\right)$ there exists $T^{*}>0$ and for any $T \in\left(0, T^{*}\right)$ there exist $V_{1 T}: \mathbb{R}^{n} \rightarrow \mathbb{R}_{>0}$ and $V_{2 T}: \mathbb{R}^{2 n} \rightarrow \mathbb{R}_{>0}$ such that for all $|\tilde{x}| \leq \Delta_{\tilde{x}},|v| \leq \Delta_{v}, T \in\left(0, T^{*}\right)$ we have the following:

$$
\begin{gathered}
\underline{\alpha}_{1}(|x|) \leq V_{1 T}(x) \leq \bar{\alpha}_{1}(|x|) \\
V_{1 T}\left(F_{T}\left(x, \phi_{T}(z), v\right)\right)-V_{1 T}(x) \\
\leq T\left(-\alpha_{1}(|x|)+\lambda_{1}(|x-z|)+\sigma_{1}(|v|)+\nu\right) \\
\underline{\alpha}_{2}(|x-z|) \leq V_{2 T}(x, z) \leq \bar{\alpha}_{2}(|x-z|) \\
V_{2 T}\left(F_{T}\left(x, \phi_{T}(z), v\right), G_{T}\left(z, h(x), \phi_{T}(z), v\right)\right)-V_{2 T}(x, z) \\
\leq T\left(-\alpha_{2}(|x-z|)+\sigma_{2}(|v|)+\nu\right)
\end{gathered}
$$

Then, there exist $\underline{\alpha}, \bar{\alpha}, \alpha \in \mathcal{K}_{\infty}$ and $\sigma \in \mathcal{G}$ such that for any triple of strictly positive real numbers $\left(\widetilde{\Delta}_{\tilde{x}}, \widetilde{\Delta}_{v}, \widetilde{\nu}\right)$ there exists $\widetilde{T}>0$ and for any $T \in(0, \widetilde{T})$ there exist $V_{T}: \mathbb{R}^{2 n} \rightarrow \mathbb{R}_{\geq 0}$ such that for all $|\tilde{x}| \leq \widetilde{\Delta}_{\tilde{x}},|v| \leq \widetilde{\Delta}_{v}$, $T \in(0, \widetilde{T})$ we have:

$$
\begin{aligned}
S P-I S S: \quad \underline{\alpha}(|\tilde{x}|) & \leq V_{T}(x, z) \leq \bar{\alpha}(|\tilde{x}|) \\
V_{T}\left(F_{T}\left(x, \phi_{T}(z), v\right),\right. & \left.G_{T}\left(z, h(x), \phi_{T}(z), v\right)\right)-V_{T}(x, z) \\
& \leq T(-\alpha(|\tilde{x}|)+\sigma(|v|)+\widetilde{\nu})
\end{aligned}
$$

Remark 5.1 There are many variations of conditions in Corollary 5.4 that could be used to state similar results (see [13,14]). Also, there is a small discrepancy between the way we write conditions in the corollary and conditions used in $[13,14]$. However, it is not hard to show that these conditions are equivalent. For example, instead of the second inequality in Corollary 5.4 we could use:

$$
\begin{aligned}
V_{1 T}\left(F_{T}\left(x, \phi_{T}(x+d), v\right)\right)-V_{1 T}(x) \\
\leq T\left(-\alpha_{1}(|x|)+\lambda_{1}(|d|)+\sigma_{1}(|v|)+\nu\right),
\end{aligned}
$$

where $d$ is a "new disturbance" (similar conditions were used in [13,14]). This condition states that the full state feedback controller $u=\phi_{T}(x)$ robustly stabilizes the plant (21) in an ISS sense. Since for the controller that uses the state estimates we can write $\phi_{T}(z)=\phi_{T}(x+(z-x))$ and let $d=x-z$, we can see that this is the same condition as the one we used in the corollary.

\section{Case study: two link manipulator}

We now revisit the problem of controlling a two link manipulator considered in [1] (see also [2]). In particular, we illustrate how Theorem 2.1 and Corollary 5.1 may be used to obtain a controller based on the Euler approximate discrete-time model of the manipulator. We emphasize that our results provide a rigorous framework for achieving ISS via approximate discrete-time models. To illustrate advantages of our approach, we compare the performance of this controller with the discretized continuous-time controller obtained in [1].

Consider a two link manipulator shown in Fig. 1, with mass of the arm $M$ and length $L$, and the gripper with mass $m$. We denote the angle of the link and the position of the gripper respectively as $\theta$ and $r$. The continuous

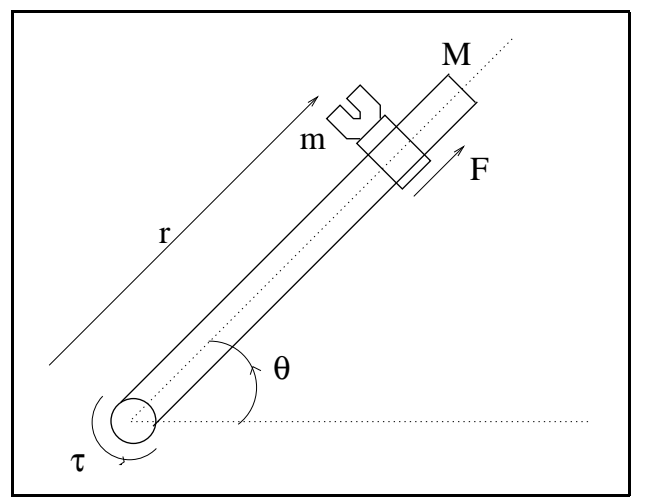

Figure 1. A two link manipulator

time model of the manipulator is:

$$
\begin{aligned}
\left(m r^{2}+M L^{2} / 3\right) \ddot{\theta}+2 m r \dot{r} \dot{\theta} & =\tau \\
m \ddot{r}-m r \dot{\theta}^{2} & =F
\end{aligned}
$$

We denote the state vector $\left(\begin{array}{llll}\theta & r & \dot{\theta} & \dot{r}\end{array}\right)^{T}$ as $x:=$ $\left(\begin{array}{llll}q_{1} & q_{2} & z_{1} & z_{2}\end{array}\right)^{T}$ and then write the state space model:

$$
\begin{aligned}
& \dot{q}_{1}=z_{1}, \quad \dot{z}_{1}=-\frac{2 m q_{2} z_{1} z_{2}}{m q_{2}^{2}+M L^{2} / 3}+\frac{\tau}{m q_{2}^{2}+M L^{2} / 3}, \\
& \dot{q}_{2}=z_{2}, \quad \dot{z}_{2}=q_{2} z_{1}^{2}+F / m,
\end{aligned}
$$


and the output equations $y_{1}=z_{1}, y_{2}=z_{2}$. The physical parameters of the manipulator and controller are as follows: $m=1 \mathrm{~kg}, M L^{2}=3 \mathrm{kgm}^{2}, k_{p_{1}}=2, k_{d_{1}}=2$, $k_{p_{2}}=1, k_{d_{2}}=1$ and $k_{n l}=1$. A continuous-time controller was designed for the system (25) in [1]:

$$
\begin{aligned}
\tau_{c}(x, w) & =-k_{d_{1}} z_{1}-k_{p_{1}}\left(q_{1}-q_{1 d}\right) \\
F_{c}(x, w) & =-k_{d_{2}} z_{2}-k_{p_{2}}\left(q_{2}-q_{2 d}\right)-k_{n l}\left(q_{2}^{3}-q_{2 d}^{3}\right),
\end{aligned}
$$

where we denoted $w:=\left(q_{1 d} q_{2 d}\right)^{T}$. This controller renders ISS for the closed-loop system (25), (26) with respect to the external inputs $q_{1 d}$ and $q_{2 d}$. Suppose now that the manipulator is controlled digitally using sample and zero order hold devices. One may simply use the controller (26) with $\tau_{c}(t)=\tau_{c}(x(k), w(k)), F_{c}(t)=$ $F_{c}(x(k), w(k)), t \in[k T,(k+1) T)$ and implement it digitally. That is, $F$ and $\tau$ are constant during sampling intervals and the state $x$ is measured at sampling instants $k T$, where $k \in \mathbb{N}$ and $T$ is the sampling period. We refer to this controller as the emulated controller (26). It was proved in $[17,36]$ that the sampled-data closedloop system with the emulated controller (26) would be semiglobally practically ISS.

However, as will be shown below, it may be better if one takes the sampling into account when designing a controller by using a discrete-time model of the plant. Since it is very hard to obtain the exact discrete-time model of the manipulator, we use instead the Euler approximate discrete-time model for the controller design. The Euler approximate model of the manipulator with sampling period $T$, when we substitute values of the physical parameters is:

$q_{1}(k+1)=q_{1}(k)+T z_{1}(k)$

$q_{2}(k+1)=q_{2}(k)+T z_{2}(k)$

$z_{1}(k+1)=z_{1}(k)+T\left[-\frac{2 q_{2}(k) z_{1}(k) z_{2}(k)}{q_{2}(k)^{2}+1}+\frac{\tau(k)}{q_{2}(k)^{2}+1}\right]$

$z_{2}(k+1)=z_{2}(k)+T\left[q_{2}(k) z_{1}(k)^{2}+F(k)\right]$.

denoted by $\widetilde{F}_{T}^{a}(x(k), \tau(k), F(k))$. In order to guarantee that the controller that achieves ISS for system (27) would also achieve SP-ISS of the sampled-data system, we need to use the results Theorem 2.1. In particular, it is directly true that consistency condition of Theorem 2.1 holds since we are using the Euler approximate model.

Controllers for sampled-data nonlinear systems often take the following form $u_{T}(x)=\sum_{i=0}^{N} T^{i} u_{i}(x)$ (see, for instance, [18] where the problem of feedback linearization was considered and [25] where backstepping based on the Euler approximate model was considered). For simplicity, we have assumed that the controller has the following form

$$
\tau_{T}^{\text {Euler }}=\tau_{c}+T u_{1}(x), \quad F_{T}^{\text {Euler }}=F_{c}+T u_{2}(x),
$$

where $u_{1}$ and $u_{2}$ are functions that need to be designed. In particular we would choose $u_{1}$ and $u_{2}$ so that we make the first difference of $V_{1 T}$ more negative. Although other controller structures and designs are possible, our choice is guided by the fact that we want to have that the continuous time and the Euler-based controllers coincide for $T=0$, so that it makes sense to compare their performance. On the other hand, we can use the freedom in choosing $u_{1}$ and $u_{2}$ in order to improve the behavior of the system. Finding a systematic controller design procedure based on these ideas is an interesting topic for further research.

We formally let the control input to be $u:=\left(u_{1} u_{2}\right)^{T}$ and using (26), (27) and (28) we can write the approximate model as follows:

$$
\begin{aligned}
& x(k+1)=\widetilde{F}_{T}^{a}\left(x(k), \tau(x(k), w(k))+T u_{1}(k),\right. \\
& \left.F(x(k), w(k))+T u_{2}(k)\right) \\
& =: \widetilde{F}_{T}^{a}(x(k), u(k), w(k)) \text {, }
\end{aligned}
$$

which has the desirable form given by (4). If $u_{1}, u_{2}$ are bounded on compact sets we can conclude that the controller (28) is locally uniformly bounded and hence the third condition of Theorem 2.1 holds.

It remains to design $u_{1}$ and $u_{2}$ so that the ISS Lyapunov conditions for approximate model in Theorem 2.1 hold. In order to do this we apply Corollary 5.1 and Remark 4.3. Let $K$ and $P$ be the kinetic and potential energy of the system $K=\frac{\left(1+q_{2}^{2}\right) z_{1}^{2}}{2}+\frac{1}{2} z_{2}^{2}, P=q_{1}^{2}+\frac{1}{2} q_{2}^{2}+\frac{1}{4} q_{2}^{4}$. In the same way as in [1], we let the Lyapunov functions $V_{1 T}$ and $V_{2 T}$ be defined as:

$$
V_{1 T}=K+P, \quad V_{2 T}=V_{1 T}+\varepsilon \frac{q_{2} z_{2}+q_{1}\left(1+q_{2}^{2}\right) z_{1}}{\left(1+q_{2}^{4}+q_{1}^{2}\right)^{3 / 4}},
$$

where $\varepsilon>0$ is a sufficiently small constant (to guarantee that $V_{2 T}$ positive definite). We next consider the first difference for $V_{1 T}$ to compute $u_{1}$ and $u_{2}$, and we write

$$
\begin{aligned}
\Delta V_{1 T}= & T\left(-2 z_{1}^{2}-z_{2}^{2}+2 z_{1} q_{1 d}+z_{2} q_{2 d}+z_{2} q_{2 d}^{3}\right) \\
& +T^{2}\left(z_{1}\left(u_{1}+3 \frac{z_{1}}{q_{2}^{2}+1}+0.5 z_{1}^{3} q_{2}^{2}\right)\right. \\
& +z_{2}\left(u_{2}+0.5 \frac{z_{2} z_{1}^{2}}{q_{2}^{2}+1}+z_{2}+1.5 z_{2} q_{2}^{2}\right) \\
& \left.+f\left(q, z, q_{d}\right)\right)+O\left(T^{3}\right),
\end{aligned}
$$

where $z:=\left(\begin{array}{ll}z_{1} & z_{2}\end{array}\right)^{T}, q:=\left(\begin{array}{ll}q_{1} & q_{2}\end{array}\right)^{T} \cdot u_{1}$ and $u_{2}$ are designed to reduce the positivity of the $O\left(T^{2}\right)$ term on the right-hand side of (30) and we choose the following:

$$
\begin{aligned}
& u_{1}(x)=-k_{e_{1}}\left(3 \frac{z_{1}}{q_{2}^{2}+1}+0.5 z_{1}^{3} q_{2}^{2}\right), \\
& u_{2}(x)=-k_{e_{2}}\left(0.5 \frac{z_{2} z_{1}^{2}}{q_{2}^{2}+1}+z_{2}+1.5 z_{2} q_{2}^{2}\right),
\end{aligned}
$$


where the values of $k_{e_{1}}=2, k_{e_{2}}=2$. Substitution of (31) to (30) results in the dissipation inequality:

$$
\begin{aligned}
\Delta V_{1 T} \leq & T\left(-\frac{1}{2}|z|^{2}+a_{1}\left|q_{1 d}\right|^{2}+a_{2}\left|q_{2 d}\right|^{6}\right) \\
& +T^{2}\left(-3 \frac{z_{1}^{2}}{q_{2}^{2}+1}-0.5 z_{1}^{4} q_{2}^{2}-0.5 \frac{z_{2}^{2} z_{1}^{2}}{q_{2}^{2}+1}-z_{2}^{2}\right. \\
& \left.-1.5 z_{2}^{2} q_{2}^{2}\right)+T^{2} f\left(q, z, q_{d}\right)+O\left(T^{3}\right),
\end{aligned}
$$

where $a_{1}$ and $a_{2}$ are sufficiently large positive numbers. The system is SP-qISS and hence the first part of condition 1 of Corollary 5.1 holds.

We now show that $V_{2 T}$ is a SP-IOSS Lyapunov function for the closed-loop approximate model

$$
\begin{aligned}
\Delta & V_{2 T}=T\left[-2 z_{1}^{2}-z_{1}^{2}+2 z_{1} q_{1 d}+z_{2} q_{2 d}+z_{2} q_{2} d^{3}\right. \\
& +\varepsilon \frac{z_{2}^{2}+2 q_{2}^{2} z_{2}^{2}+z_{1}^{2}+q_{2}\left(F_{c}+T u_{2}\right)+q_{1}\left(\tau_{c}+T u_{1}\right)}{\left(1+q_{2}^{4}+q_{1}^{2}\right)^{3 / 4}} \\
& \left.+\frac{3}{4} \varepsilon \frac{4 q_{2}^{3} z_{2}+2 q_{1} z_{1}}{\left(1+q_{2}^{4}+q_{1}^{2}\right)^{7 / 4}}\left(q_{2} z_{2}+q_{1}\left(1+q_{2}^{2}\right) z_{1}\right)\right]+O\left(T^{2}\right) \\
\leq & T\left[M_{1}\left(q_{1 d}^{2}+q_{2 d}^{2}+q_{2 d}^{6}\right)-M_{2}|z|^{2}+M_{3}|z|^{2}\right. \\
& \left.+\varepsilon \frac{q_{2} F_{c}+q_{1} \tau_{c}}{\left(1+q_{2}^{4}+q_{1}^{2}\right)^{3 / 4}}\right]+O\left(T^{2}\right),
\end{aligned}
$$

for sufficiently small $T, \varepsilon$ and $M_{2}$ and sufficiently large $M_{1}$ and $M_{3}$. Substituting the controller $\tau_{T}^{\text {Euler }}$ and $F_{T}^{E u l e r}$, we can write the dissipation inequality as

$$
\begin{aligned}
\Delta V_{2 T} \leq & T\left\{\tilde{M}_{1}\left(q_{1 d}^{2}+q_{2 d}^{2}+q_{2 d}^{6}\right)+\tilde{M}_{3}|z|^{2}\right. \\
& \left.-\tilde{M}_{2}|z|^{2}-\tilde{\varepsilon} \frac{q_{2}^{4}+q_{1}^{2}}{\left(1+q_{2}^{4}+q_{1}^{2}\right)^{3 / 4}}\right\}+O\left(T^{2}\right),
\end{aligned}
$$

for sufficiently small $T, \tilde{\varepsilon}$ and $\tilde{M}_{2}$ and sufficiently large $\tilde{M}_{1}$ and $\tilde{M}_{3}$. The system is SP-IOSS and hence the second part of condition 1 of Corollary 5.1 holds. Finally, since $\alpha_{1}(s)=\frac{s^{2}}{2}$ and $\lambda_{2}(s)=\tilde{M}_{3} s^{2}$, it is obvious that condition 2 of Corollary 5.1 holds. From Corollary 5.1 and Remark 4.3 we have that the closed-loop approximate model (27), (28) is SP-ISS, and from the choice of $V_{1 T}$ and $V_{2 T}$ the first condition of Theorem 2.1 holds. Hence, we have that all conditions of Theorem 2.1 are satisfied. Then, it follows from the conclusion of the theorem that the exact discrete-time closed-loop system is SP-ISS. Finally, using results of [24] we conclude that the closed-loop sampled-data system (25), (28) is SP-ISS.

We present simulation results to illustrate performance of the system when we apply the Euler-based controller (28) and the emulated controller (26). Simulation were carried out using SIMULINK with the following simulation parameters $T=0.25 s, x_{\circ}=\left(\begin{array}{llll}0.1 & 0.1 & 0.1 & 0.1\end{array}\right)^{T}$, $\theta_{d}(t)=3$ square $(0.5 t)$ and $r_{d}(t)=0$. The results are presented in Fig. 2. Fig. 2(a) shows the reference signal $\theta_{d}$ and the actual angular position of the $\operatorname{arm} \theta$, while Fig. 2(b) shows the desired position of the gripper $r_{d}$ and the actual position $r$ obtained when applying the
Euler-based controller (28). Fig. 2(c) and Fig. 2(d) are respectively showing the response of the corresponding variables with emulated controller (26). The simulation is carried out with a relatively large sampling period, to observe the robustness of each controller to a square wave input. It is shown that with the given simulation set-up, Euler-based controller can still show a good performance with $T=0.25 \mathrm{~s}$. On the other hand, the trajectories of the system with the emulated controller (26), exhibit finite escape times for the same simulation parameters. Moreover, it is shown in Fig. 2(d) and Fig. 2 (e) that by reducing the time sampling into $T=0.1 s$, the emulation controller results in a bounded response, although the overshoot that occurs on the state $r$ exceeds the feasible range of the physical parameters of the manipulator. Reducing $T$ further results in performance that is closer to the continuous-time controller.
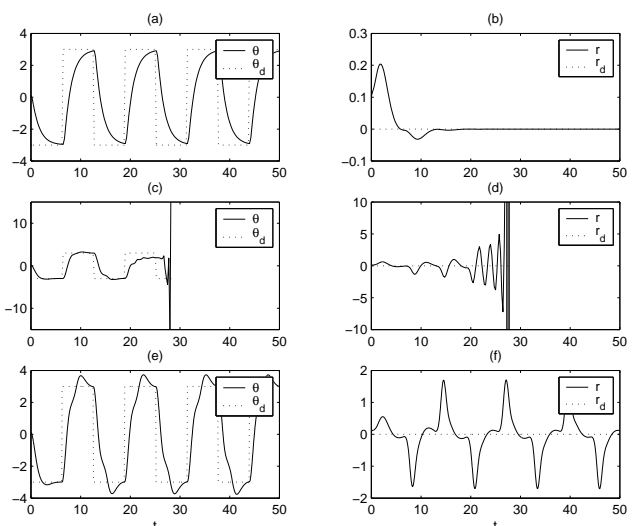

Figure 2. Responses with Euler-based controller (a,b) and emulation controller $(c, d, e, f)$ to a square wave input.

\section{Conclusions}

We have presented results on changing supply rates for discrete-time SP-IOSS systems that allow for a partial construction of Lyapunov functions for parameterized discrete-time systems. We have applied our results to several problems and a case study. The results play an important role and provide a strong motivation for the development of systematic controller design procedures for sampled-data nonlinear systems based on their approximate discrete-time models.

\section{Acknowledgements}

The authors wish to thank David Angeli for his useful comments and suggestions. This research was supported by the Australian Research Council under the Large Grants Scheme.

\section{References}

[1] D. Angeli. Input-to-state stability of PD-controlled robotic systems. Automatica, 35:1285-1290, 1999. 
[2] D. Angeli, E. D. Sontag, and Y. Wang. A characterization of integral input to state stability. IEEE Trans. Auto. Contr., 45:1082-1097, 2000

[3] M. Arcak, D. Angeli, and E.D. Sontag. A unifying integral ISS framework for stability of nonlinear cascades. SIAM J. Optimiz. Contr., 40:1888-1904, 2002.

[4] J.P. Barbot, S. Monaco, and D. Normand-Cyrot. A sampled normal form for feedback linearization. Math. Contr. Sig. Sys., 9:162-188, 1996.

[5] M. Bensoubaya, A. Ferfera, and A. Iggidr. Stabilization of nonlinear systems by use of semidefinite lyapunov functions. Appl. Math. Letters, 12:11-17, 1999.

[6] D. Dochain and G. Bastin. Adaptive identification and control algorithms for nonlinear bacterial growth systems. Automatica, 20:621-634, 1984.

[7] F. Esfandiari and H. K. Khalil. On the robustness of sampleddata control to unmodelled high frequency dynamics. IEEE Trans. Auto. Contr., 34:900-903, 1989.

[8] G. C. Goodwin, B. McInnis, and R. S. Long. Adaptive control algorithm for waste water treatment and ph neutralization. Optimal Contr. Applic. Meth., 3:443-459, 1982.

[9] J.W. Grizzle and J.-M. Kang. Discrete-time control design with positive semidefinite lyapunov functions. Syst. Contr. Lett., 43:287-292, 2001

[10] A. Isidori. Nonlinear Control Systems II. Springer, 1999.

[11] Z.P. Jiang, I.M.Y. Mareels, and Y. Wang. A lyapunov formulation of the nonlinear small gain theorem for interconnected ISS systems. Automatica, 32:1211-1215, 1996.

[12] Z.P. Jiang and Y. Wang. Input-to-state stability for discretetime nonlinear systems. Automatica, 37:857-869, 2001.

[13] D. Kazakos and J. Tsinias. Stabilization of nonlinear discretetime systems using state detection. IEEE Trans. Auto. Contr., 38:1398-1400, 1993.

[14] D. Kazakos and J. Tsinias. The input to state stability condition and global stabilization of discrete-time systems. IEEE Trans. Auto. Contr., 39:2111-2113, 1994.

[15] H.K. Khalil. Nonlinear Control Systems 2nd Ed. Prentice Hall, 1996.

[16] M. Krstić, I. Kanellakopoulos, and P. V. Kokotović. Nonlinear and Adaptive Control Design. Wiley, 1995.

[17] D. S. Laila, D. Nešić, and A. R. Teel. Open and closed loop dissipation inequalities under sampling and controller emulation. European Journal of Control, 8:109-125, 2002.

[18] H. G. Lee, A. Araposthatis, and S. I. Marcus. On the digital control of nonlinear systems. In Proc. IEEE Conf. Decis. Contr., volume 1, pages 480-481, 1998.

[19] I. M. Y. Mareels, H. B. Penfold, and R. J. Evans. Controlling nonlinear time-varying systems via euler approximations. Automatica, 28:681-696, 1992.

[20] S. Monaco and D. Normand-Cyrot. Sampling of a linear analytic control system. In Proc. IEEE Conf. Decis. Contr., pages $1457-1462,1985$.

[21] D. Nešić and D. Angeli. Integral versions of ISS for sampleddata nonlinear systems via their approximate discrete-time models. to appear in IEEE Trans. Auto. Contr., 47, 2002.

[22] D. Nešić and D. S. Laila. A note on input to state stabilization for nonlinear sampled-data systems. IEEE Trans. Auto. Contr., 47:1153-1158, 2002.

[23] D. Nešić and A. R. Teel. A framework for stabilization of nonlinear sampled-data systems based on their approximate discrete-time models. accepted in IEEE Trans. Auto. Contr., 2002 .
[24] D. Nešić, A. R. Teel, and E.D. Sontag. Formulas relating $\mathcal{K} \mathcal{L}$ stability estimates of discrete-time and sampled-data nonlinear systems. Syst. Contr. Lett., 38:49-60, 1999.

[25] D. Nešić and A.R. Teel. Backstepping on the Euler approximate model for stabilization of sampled-data nonlinear systems. In Proc. IEEE Conf. Decis. Contr., pages 1737-1742, Orlando, FL, 2001.

[26] D. Nešić and A.R. Teel. Changing supply functions in input to state stable systems: The discrete-time case. IEEE Trans. Auto. Contr., 45:960-962, 2001.

[27] D. Nešić, A.R. Teel, and P. Kokotović. Sufficient conditions for stabilization of sampled-data nonlinear systems via discrete-time approximations. Syst. Contr. Lett., 38:259-270, 1999.

[28] N. Rouche, P. Habets, and M. Laloy. Stability Theory by Lyapunov's Direct Method. Springer, London, 1977.

[29] E. Skafidas, A. Fradkov, R. J. Evans, and I. M. Y. Mareels. Trajectory based adaptive control for nonlinear systems under matching conditions. Automatica, 34:287-299, 1998.

[30] E. D. Sontag. The ISS philosophy as a unifying framework for stability-like behaviour. In A. Isidori, F. LamnabhiLagarrigue, and W. Respondek, editors, Nonlinear Control in the Year 2000, Lecture Notes in Control and Information Sciences, volume 2, pages 443-468. Springer, Berlin, 2000.

[31] E. D. Sontag and A.R. Teel. Changing supply functions in input to state stable systems. IEEE Trans. Auto. Contr., 40:1476-1478, 1995.

[32] E. D. Sontag and Y. Wang. Output to state stability and detectability of nonlinear systems. SCL, 29:279-290, 1997.

[33] E.D. Sontag. Smooth stabilization implies coprime factorization. IEEE Trans. Auto. Contr., 34:435-443, 1989.

[34] A. M. Stuart and A. R. Humphries. Dynamical systems and numerical analysis. Cambridge Univ. Press, New York, 1996.

[35] A. R. Teel, L. Moreau, and D. Nešić. A unification of timescale methods for systems with disturbances. to appear in IEEE Trans. Auto. Contr., 2002.

[36] A.R. Teel, D. Nešić, and P. Kokotović. A note on ISS of nonlinear sampled-data systems. In Proc. IEEE Conf. Decis. Contr, pages 2473-2478, Tampa, FL, 1998.

[37] L. Zaccarian, A.R. Teel, and D. Nešić. On finite gain $L_{p}$ stability of nonlinear sampled-data systems. to appear in Syst. Contr. Lett., 2002.

\section{A Proofs of main results}

The following remark, together with Lemmas 1 and 2 of [31], is used in proving Theorems 4.1 and 4.2 .

Remark A.1 Since for any $\alpha \in \mathcal{K}$ we have $\alpha\left(s_{1}+\right.$ $\left.s_{2}\right) \leq \alpha\left(2 s_{1}\right)+\alpha\left(2 s_{2}\right)$ for all $s_{1} \geq 0, s_{2} \geq 0$, then for any $\alpha_{1}, \alpha_{2} \in \mathcal{K}$, there exist $\underline{\alpha}, \bar{\alpha} \in \mathcal{K}$ such that $\underline{\alpha}\left(s_{1}+s_{2}\right) \leq \alpha_{1}\left(s_{1}\right)+\alpha_{2}\left(s_{2}\right) \leq \bar{\alpha}\left(s_{1}+s_{2}\right)$, for all $s_{1} \geq 0, s_{2} \geq 0$, where $\underline{\alpha}(s):=\min \left\{\alpha_{1}\left(\frac{s}{2}\right), \alpha_{2}\left(\frac{s}{2}\right)\right\}$ and $\bar{\alpha}(s):=2 \max \left\{\alpha_{1}(s), \alpha_{2}(s)\right\}$.

Proof of Lemma 4.1: We denote $V_{T}\left(F_{T}\right):=V_{T}\left(F_{T}(x, u)\right)$ and $V_{T}:=V_{T}(x)$. Suppose that all conditions in Lemma 4.1 are satisfied. Fix an arbitrary $q \in \mathcal{S N}$ and let $\rho$ be defined using (13). We prove next that $\rho\left(V_{T}\right)$ is a SP-IOSS Lyapunov function for the system with appropriate bounding and measuring functions stated in the 
lemma. From the Mean Value Theorem and the fact that $q(\cdot)=\frac{d \rho}{d s}(\cdot)$ is nondecreasing we have:

$$
\rho(a)-\rho(b) \leq q(a)[a-b] \quad \forall a \geq 0, b \geq 0 .
$$

Let arbitrary strictly positive real numbers $\left(\Delta_{x}^{\prime}, \Delta_{u}^{\prime}, \nu^{\prime}\right)$ be given. Let $\Delta_{x}^{\prime}, \Delta_{u}^{\prime}$ generate numbers $M, T_{1}^{*}$ via the third condition of the lemma, so that (14) holds. Let $\nu_{1}$ be such that $\max \{\lambda(M), \sigma(M)\}\left[q\left(s+\nu_{1}\right)-q(s)\right] \leq$ $\frac{\nu^{\prime}}{2}, \forall s \in[0, \bar{\alpha}(M)+2 \max \{\lambda(M), \sigma(M)\}]$. Such $\nu_{1}$ always exists since $q(\cdot)$ is continuous.

We define $\Delta_{x}:=\Delta_{x}^{\prime}, \Delta_{u}:=\Delta_{u}^{\prime}, \nu:=\min \left\{\frac{\nu^{\prime}}{2 q \circ \bar{\alpha}(M)}, \nu_{1}\right\}$. Let $\left(\Delta_{x}, \Delta_{u}, \nu\right)$ determine $T_{2}^{*}>0$ and $V_{T}$ using the first condition of the lemma, such that for all $T \in\left(0, T_{2}^{*}\right)$ and all $\left|w_{x}(x)\right| \leq \Delta_{x},\left|w_{u}(u)\right| \leq \Delta_{u}$ the inequalities (10) and (11) hold. Fix $T^{*}:=\min \left\{T_{1}^{*}, T_{2}^{*}, 1\right\}$. In the rest of the proof we always consider arbitrary $T \in\left(0, T^{*}\right)$, $\left|w_{x}(x)\right| \leq \Delta_{x}$ and $\left|w_{u}(u)\right| \leq \Delta_{u}$.

Note that a direct consequence of condition 1 of the lemma and the fact that $T^{*} \leq 1$ is:

$$
\begin{gathered}
V_{T} \geq \max \left\{\underline{\alpha}\left(\left|w_{\underline{\alpha}}(x)\right|\right), T \alpha\left(\left|w_{\alpha}(x)\right|\right)\right. \\
\left.-T \lambda\left(\left|w_{\lambda}(x)\right|\right)-T \sigma\left(\left|w_{\sigma}(u)\right|\right)-T \nu\right\} \\
V_{T}\left(F_{T}\right) \leq \\
\quad \bar{\alpha}\left(\left|w_{\alpha}(x)\right|\right)+\lambda\left(\left|w_{\lambda}(x)\right|\right) \\
+\sigma\left(\left|w_{\sigma}(u)\right|\right)+\nu .
\end{gathered}
$$

Note first that $\rho \circ \underline{\alpha}\left(\left|w_{\underline{\alpha}}(x)\right|\right) \leq \rho\left(V_{T}\right) \leq \rho \circ \bar{\alpha}\left(\left|w_{\bar{\alpha}}(x)\right|\right)$, which shows that (10) holds with the new bounding functions $\underline{\alpha}^{\prime}(s)=\rho \circ \underline{\alpha}(s)$ and $\bar{\alpha}^{\prime}(s)=\rho \circ \bar{\alpha}(s)$ and the same measuring functions. Now we prove that (11) holds for $\rho\left(V_{T}\right)$ with the new bounding functions and the same measuring functions. The following two preliminary cases are first considered:

1. $V_{T}\left(F_{T}\right) \leq \frac{1}{2} V_{T}$ Using the inequalities (A.1) and (A.2) and the definition of $M$ and $\nu$ we obtain

$$
\begin{aligned}
\rho( & \left.V_{T}\left(F_{T}\right)\right)-\rho\left(V_{T}\right) \\
\leq & \rho\left(\frac{1}{2} V_{T}\right)-\rho\left(V_{T}\right) \leq q\left(\frac{1}{2} V_{T}\right)\left[-\frac{1}{2} V_{T}\right] \\
\leq & \frac{T}{2} q\left(\frac{1}{2} V_{T}\right) \cdot\left(-\alpha\left(\left|w_{\alpha}(x)\right|\right)+\lambda\left(\left|w_{\lambda}(x)\right|\right)\right. \\
& \left.+\sigma\left(\left|w_{\sigma}(u)\right|\right)+\nu\right) \\
\leq & \frac{T}{2} q\left(\frac{1}{2} V_{T}\right) \cdot\left(-\alpha\left(\left|w_{\alpha}(x)\right|\right)+\lambda\left(\left|w_{\lambda}(x)\right|\right)\right. \\
& \left.+\sigma\left(\left|w_{\sigma}(u)\right|\right)\right)+T \frac{q \circ \bar{\alpha}(M)}{2} \nu \\
\leq & \frac{T}{2} q\left(\frac{1}{2} V_{T}\right) \cdot\left(-\alpha\left(\left|w_{\alpha}(x)\right|\right)+\lambda\left(\left|w_{\lambda}(x)\right|\right)\right. \\
& \left.+\sigma\left(\left|w_{\sigma}(u)\right|\right)\right)+T \frac{\nu^{\prime}}{4}
\end{aligned}
$$

2. $V_{T}\left(F_{T}\right)>\frac{1}{2} V_{T}$ Using the inequalities (A.1) and (11) and the definition of $M$ and $\nu$ we obtain

$$
\begin{aligned}
\rho( & \left.V_{T}\left(F_{T}\right)\right)-\rho\left(V_{T}\right) \\
\leq & q\left(V_{T}\left(F_{T}\right)\right)\left[V_{T}\left(F_{T}\right)-V_{T}\right] \\
\leq & T q\left(V_{T}\left(F_{T}\right)\right) \cdot\left(-\alpha\left(\left|w_{\alpha}(x)\right|\right)+\lambda\left(\left|w_{\lambda}(x)\right|\right)\right. \\
& \left.+\sigma\left(\left|w_{\sigma}(u)\right|\right)+\nu\right) \\
\leq & T q\left(V_{T}\left(F_{T}\right)\right) \cdot\left(-\alpha\left(\left|w_{\alpha}(x)\right|\right)+\lambda\left(\left|w_{\lambda}(x)\right|\right)\right. \\
& \left.+\sigma\left(\left|w_{\sigma}(u)\right|\right)\right)+T q \circ \bar{\alpha}(M) \nu \\
\leq & T q\left(V_{T}\left(F_{T}\right)\right) \cdot\left(-\alpha\left(\left|w_{\alpha}(x)\right|\right)+\lambda\left(\left|w_{\lambda}(x)\right|\right)\right. \\
& \left.+\sigma\left(\left|w_{\sigma}(u)\right|\right)\right)+T \frac{\nu^{\prime}}{2} .
\end{aligned}
$$

The proof is completed by considering the following three cases:

Case 1: $\lambda\left(\left|w_{\lambda}(x)\right|\right)+\sigma\left(\left|w_{\sigma}(u)\right|\right) \leq \frac{1}{2} \alpha\left(\left|w_{\alpha}(x)\right|\right)$

- $V_{T}\left(F_{T}\right) \leq \frac{1}{2} V_{T}$ We use (A.4) to write:

$$
\begin{aligned}
\rho\left(V_{T}\left(F_{T}\right)\right)-\rho\left(V_{T}\right) & \leq \frac{T}{2} q\left(\frac{1}{2} V_{T}\right) \cdot\left(-\frac{1}{2} \alpha\left(\left|w_{\alpha}(x)\right|\right)\right)+T \frac{\nu^{\prime}}{4} \\
& \leq-\frac{T}{4} q\left(\frac{1}{2} V_{T}\right) \cdot \alpha\left(\left|w_{\alpha}(x)\right|\right)+T \frac{\nu^{\prime}}{4}
\end{aligned}
$$

- $V_{T}\left(F_{T}\right)>\frac{1}{2} V_{T}$ We use (A.6) and the fact that $q$ is nondecreasing to write:

$$
\begin{gathered}
\rho\left(V_{T}\left(F_{T}\right)\right)-\rho\left(V_{T}\right) \leq T q\left(V_{T}\left(F_{T}\right)\right) \cdot\left(-\frac{1}{2} \alpha\left(\left|w_{\alpha}(x)\right|\right)\right) \\
+T \frac{\nu^{\prime}}{2} \leq-\frac{T}{4} q\left(\frac{1}{2} V_{T}\right) \cdot \alpha\left(\left|w_{\alpha}(x)\right|\right)+T \frac{\nu^{\prime}}{2}
\end{gathered}
$$

Since $q$ is nondecreasing, using (A.2) and the second condition of the lemma, the following holds for Case 1 :

$$
\begin{aligned}
& \rho\left(V_{T}\left(F_{T}\right)\right)-\rho\left(V_{T}\right) \\
\leq & -\frac{T}{4} q\left(\frac{1}{2} \underline{\alpha} \circ \underline{\kappa}\left(\left|w_{\alpha}(x)\right|\right)\right) \cdot \alpha\left(\left|w_{\alpha}(x)\right|\right)+T \frac{\nu^{\prime}}{2}
\end{aligned}
$$

Case $2: \lambda\left(\left|w_{\lambda}(x)\right|\right)+\sigma\left(\left|w_{\sigma}(u)\right|\right)>\frac{1}{2} \alpha\left(\left|w_{\alpha}(x)\right|\right)$, $\overline{\lambda\left(\left|w_{\lambda}(x)\right|\right) \geq \sigma\left(\left|w_{\sigma}(u)\right|\right)}$

- $V_{T}\left(F_{T}\right) \leq \frac{1}{2} V_{T}$ We use (A.4), (10), the fact that $q$ is nondecreasing, $T^{*} \leq 1$ and the choice of $\nu_{1}$ to write:

$$
\begin{aligned}
& \rho\left(V_{T}\left(F_{T}\right)\right)-\rho\left(V_{T}\right) \\
& \leq \frac{T}{2} q\left(\frac{1}{2} V_{T}\right) \cdot\left(-\alpha\left(\left|w_{\alpha}(x)\right|\right)+2 \lambda\left(\left|w_{\lambda}(x)\right|\right)\right)+T \frac{\nu^{\prime}}{4} \\
& \leq-\frac{T}{2} q\left(\frac{1}{2} V_{T}\right) \cdot \alpha\left(\left|w_{\alpha}(x)\right|\right)+T q\left(\frac{1}{2} \bar{\alpha}\left(\left|w_{\bar{\alpha}}(x)\right|\right)\right) \\
& \lambda\left(\left|w_{\lambda}(x)\right|\right)+T \frac{\nu^{\prime}}{4} \\
& \leq-\frac{T}{2} q\left(\frac{1}{2} V_{T}\right) \cdot \alpha\left(\left|w_{\alpha}(x)\right|\right)+T q\left(\bar{\alpha}\left(\left|w_{\bar{\alpha}}(x)\right|\right)\right. \\
&\left.+2 \lambda\left(\left|w_{\lambda}(x)\right|\right)\right) \cdot \lambda\left(\left|w_{\lambda}(x)\right|\right)+T \frac{\nu^{\prime}}{2}+T \frac{\nu^{\prime}}{4}
\end{aligned}
$$


- $V_{T}\left(F_{T}\right)>\frac{1}{2} V_{T}$ We use (A.6), (10), the fact that $q$ is nondecreasing, $T^{*} \leq 1$ and the choice of $\nu_{1}$ to write:

$$
\begin{aligned}
\rho( & \left.V_{T}\left(F_{T}\right)\right)-\rho\left(V_{T}\right) \\
\leq & T q\left(V_{T}\left(F_{T}\right)\right) \cdot\left(-\alpha\left(\left|w_{\alpha}(x)\right|\right)+2 \lambda\left(\left|w_{\lambda}(x)\right|\right)\right)+T \frac{\nu^{\prime}}{2} \\
\leq & -T q\left(\frac{1}{2} V_{T}\right) \cdot \alpha\left(\left|w_{\alpha}(x)\right|\right)+2 T q\left(\bar{\alpha}\left(\left|w_{\bar{\alpha}}(x)\right|\right)\right. \\
& \left.+2 \lambda\left(\left|w_{\lambda}(x)\right|\right)+\nu_{1}\right) \cdot \lambda\left(\left|w_{\lambda}(x)\right|\right)+T \frac{\nu^{\prime}}{2} \\
\leq & -T q\left(\frac{1}{2} V_{T}\right) \cdot \alpha\left(\left|w_{\alpha}(x)\right|\right)+2 T q\left(\bar{\alpha}\left(\left|w_{\bar{\alpha}}(x)\right|\right)\right. \\
& \left.+2 \lambda\left(\left|w_{\lambda}(x)\right|\right)\right) \cdot \lambda\left(\left|w_{\lambda}(x)\right|\right)+T \frac{\nu^{\prime}}{2}+T \frac{\nu^{\prime}}{2}
\end{aligned}
$$

Since $q$ is nondecreasing, using (A.2), (A.8), (A.9), the second condition of the lemma, the condition that $\lambda\left(\left|w_{\lambda}(x)\right|\right)>\frac{1}{4} \alpha\left(\left|w_{\alpha}(x)\right|\right)$ and the definition of $\theta_{\lambda}$, the following always holds for Case 2 :

$$
\begin{aligned}
\rho\left(V_{T}\left(F_{T}\right)\right)-\rho\left(V_{T}\right) \\
\leq-\frac{T}{2} q\left(\frac{1}{2} \underline{\alpha} \circ \underline{\kappa}\left(\left|w_{\alpha}(x)\right|\right)\right) \cdot \alpha\left(\left|w_{\alpha}(x)\right|\right) \\
\quad+2 T q \circ \theta_{\lambda}\left(\left|w_{\lambda}(x)\right|\right) \cdot \lambda\left(\left|w_{\lambda}(x)\right|\right)+T \nu^{\prime}
\end{aligned}
$$

Case 3: $\lambda\left(\left|w_{\lambda}(x)\right|\right)+\sigma\left(\left|w_{\sigma}(u)\right|\right)>\frac{1}{2} \alpha\left(\left|w_{\alpha}(x)\right|\right)$, $\overline{\lambda\left(\left|w_{\lambda}(x)\right|\right)<\sigma\left(\left|w_{\sigma}(u)\right|\right)}$

Following a similar way as in Case 2, the following always holds for Case 3:

$$
\begin{aligned}
& \rho\left(V_{T}\left(F_{T}\right)\right)-\rho\left(V_{T}\right) \\
& \quad \leq-\frac{T}{2} q\left(\frac{1}{2} \underline{\alpha} \circ \underline{\kappa}\left(\left|w_{\alpha}(x)\right|\right)\right) \cdot \alpha\left(\left|w_{\alpha}(x)\right|\right) \\
& \quad+2 T q \circ \theta_{\sigma}\left(\left|w_{\sigma}(u)\right|\right) \cdot \sigma\left(\left|w_{\sigma}(u)\right|\right)+T \nu^{\prime}
\end{aligned}
$$

We have shown through these three cases that the following holds:

$$
\begin{aligned}
& \rho\left(V_{T}\left(F_{T}\right)\right)-\rho\left(V_{T}(x)\right) \\
& \leq T\left[-\frac{1}{4} q \circ \frac{1}{2} \underline{\alpha} \circ \underline{\kappa}\left(\left|w_{\alpha}(x)\right|\right) \cdot \alpha\left(\left|w_{\alpha}(x)\right|\right)\right. \\
& \quad+2 q \circ \theta_{\lambda}\left(\left|w_{\lambda}(x)\right|\right) \cdot \lambda\left(\left|w_{\lambda}(x)\right|\right) \\
& \left.\quad+2 q \circ \theta_{\sigma}\left(\left|w_{\sigma}(u)\right|\right) \cdot \sigma\left(\left|w_{\sigma}(u)\right|\right)+\nu^{\prime}\right],
\end{aligned}
$$

which completes the proof of Lemma 4.1 .

Proof of Theorem 4.1: Suppose that all conditions of the theorem be satisfied. Let $\underline{\alpha}_{1}, \bar{\alpha}_{1}, \alpha_{1}, \sigma_{1}$ come from the condition 1 and $\underline{\alpha}_{2}, \bar{\alpha}_{2}, \alpha_{2}, \lambda_{2}, \sigma_{2}$ come from the condition 2. Define $\tilde{q}$ as:

$$
\tilde{q}(r):=\inf _{r \leq s} \frac{\alpha_{1} \circ \gamma_{1}^{-1}(s)}{2\left(1+\lambda_{2}(s)\right)},
$$

where $\gamma_{1}$ comes from the third condition of the theorem. Notice that $\tilde{q}$ is by definition a nondecreasing function. Condition 4 of the theorem implies $\tilde{q}(r)>0$ for all $r>0$. Let $q(s):=\tilde{q} \circ \theta_{\lambda_{2}}^{-1}(s)$, where $\theta_{\lambda_{2}}$ is defined in Lemma 4.1. Using $q(\cdot)$ we define $\rho(\cdot)$ via (13). Let $\rho$ generate via Lemma 4.1 the new bounding functions $\underline{\alpha}_{2}^{\prime}, \bar{\alpha}_{2}^{\prime}, \alpha_{2}^{\prime}, \lambda_{2}^{\prime}$, $\sigma_{2}^{\prime}$

Let arbitrary strictly positive real numbers $\left(\Delta_{x}, \Delta_{u}, \nu\right)$ be given. Let $\left(\Delta_{x}, \Delta_{u}, \frac{\nu}{2}\right)$ generate via condition 1 the number $T_{1}^{*}$ and $V_{1 T}$. Let $\left(\gamma_{2}\left(\Delta_{x}\right), \gamma_{3}\left(\Delta_{u}\right), \frac{\nu}{2}\right)$ generate via condition 2 and Lemma 4.1 the number $T_{2}^{*}$ and $\rho\left(V_{2 T}\right)$. Let $T^{*}=\min \left\{T_{1}^{*}, T_{2}^{*}\right\}$ and define now $V_{T}$ as (15). Let $w_{x}(x):=w_{x_{1}}(x)$ and $w_{u}(u):=w_{u_{1}}(u)$. We consider now arbitrary $\left|w_{x}(x)\right| \leq \Delta_{x},\left|w_{u}(u)\right| \leq \Delta_{u}$ and $T \in\left(0, T^{*}\right)$. Note that this implies via condition 3 of the theorem that $w_{x_{2}}(x) \leq \gamma_{2}\left(\Delta_{x}\right)$ and $w_{u_{2}}(x) \leq \gamma_{3}\left(\Delta_{u}\right)$.

First, it follows from the definition of $V_{T}$ that

$$
\begin{aligned}
& \underline{\alpha}_{1}\left(\left|w_{\underline{\alpha}_{1}}(x)\right|\right)+\rho \circ \underline{\alpha}_{2}\left(\left|w_{\underline{\alpha}_{2}}(x)\right|\right) \leq V_{T}(x) \\
& \leq \bar{\alpha}_{1}\left(\left|w_{\bar{\alpha}_{1}}(x)\right|\right)+\rho \circ \bar{\alpha}_{2}\left(\left|w_{\bar{\alpha}_{2}}(x)\right|\right) .
\end{aligned}
$$

Then by Remark A.1, there exist $\underline{\alpha}, \bar{\alpha} \in \mathcal{K}_{\infty}$ such that

$$
\begin{array}{r}
\underline{\alpha}\left(\left|w_{\underline{\alpha}_{1}}(x)\right|+\left|w_{\underline{\alpha}_{2}}(x)\right|\right) \leq V_{T}(x) \\
\leq \bar{\alpha}\left(\left|w_{\bar{\alpha}_{1}}(x)\right|+\left|w_{\bar{\alpha}_{2}}(x)\right|\right) .
\end{array}
$$

Using condition 4 of the theorem, the dissipation inequality for $V_{T}$ can be written as:

$$
\begin{aligned}
& V_{T}\left(F_{T}\right)-V_{T}(x) \\
& =V_{1 T}\left(F_{T}\right)-V_{1 T}+\rho\left(V_{2 T}\left(F_{T}\right)\right)-\rho\left(V_{2 T}\right) \\
& \leq T\left[\sigma_{1}\left(\left|w_{\sigma_{1}}(u)\right|\right)+\sigma_{2}^{\prime}\left(\left|w_{\sigma_{2}}(u)\right|\right)+\frac{\nu}{2}-\alpha_{1}\left(\left|w_{\alpha_{1}}(x)\right|\right)\right. \\
& \left.\quad+\lambda_{2}^{\prime} \circ \gamma_{1}\left(\left|w_{\alpha_{1}}(x)\right|\right)-\alpha_{2}^{\prime}\left(\left|w_{\alpha_{2}}(x)\right|\right)+\frac{\nu}{2}\right] \\
& \leq T\left[\sigma_{1}\left(\left|w_{\sigma_{1}}(u)\right|\right)+\sigma_{2}^{\prime}\left(\left|w_{\sigma_{2}}(u)\right|\right)+\frac{\nu}{2}-\alpha_{1}\left(\left|w_{\alpha_{1}}(x)\right|\right)\right. \\
& \left.\quad+\frac{\alpha_{1}\left(\left|w_{\alpha_{1}}(x)\right|\right) \lambda_{2} \circ \gamma_{1}\left(\left|w_{\alpha_{1}}(x)\right|\right)}{2\left(1+\lambda_{2} \circ \gamma_{1}\left(\left|w_{\alpha_{1}}(x)\right|\right)\right)}-\alpha_{2}^{\prime}\left(\left|w_{\alpha_{2}}(x)\right|\right)+\frac{\nu}{2}\right] .
\end{aligned}
$$

Since $\frac{\lambda_{2}(s)}{1+\lambda_{2}(s)} \leq 1, \forall s \geq 0$, by monotonicity of $q(\cdot)$ and using Remark A.1, there exist $\alpha \in \mathcal{K}_{\infty}$ and $\sigma \in \mathcal{K}$ so that we can write

$$
\begin{aligned}
& V_{T}\left(F_{T}\right)-V_{T}(x) \leq-T \alpha\left(\left|w_{\alpha_{1}}(x)\right|+\left|w_{\alpha_{2}}(x)\right|\right) \\
& \quad+T \sigma\left(\left|w_{\sigma_{1}}(u)\right|+\left|w_{\sigma_{2}}(u)\right|\right)+T \nu
\end{aligned}
$$

This completes the proof of Theorem 4.1.

Proof of Theorem 4.2: Suppose that all conditions of the theorem are satisfied. Let $\underline{\alpha}_{1}, \bar{\alpha}_{1}, \alpha_{1}, \sigma_{1}$ come from the condition 1 and $\underline{\alpha}_{2}, \bar{\alpha}_{2}, \alpha_{2}, \lambda_{2}, \sigma_{2}$ come from the 
condition 2. Define a function $\alpha_{1}^{\prime} \in \mathcal{K}_{\infty}$ as follows

$$
\alpha_{1}^{\prime}(s):= \begin{cases}\alpha_{1}(s) & \text { for small } s \\ \lambda_{2} \circ \gamma_{1}(s) & \text { for large } s\end{cases}
$$

where $\gamma_{1}$ comes from the third condition of the theorem. It is clear that $\alpha_{1}^{\prime}(s)=O\left[\alpha_{1}(s)\right]$ for $s \rightarrow 0^{+}$. Hence, by Lemma 2 of [31], there exists $\tilde{q}_{1} \in \mathcal{S} N$ such that $\tilde{q}_{1}(s) \cdot \alpha_{1}(s) \geq \alpha_{1}^{\prime}(s)$. Further, define a function $\lambda_{2}^{\prime}(s):=$ $\frac{1}{2} \alpha_{1}^{\prime} \circ \gamma_{1}^{-1}(s)$ and note that $\lambda_{2}^{\prime} \in \mathcal{K}$ and it is clear that $\lambda_{2}(s)=O\left[\lambda_{2}^{\prime}(s)\right]$ for $s \rightarrow+\infty$. Then by Lemma 1 of [31], there exists $\tilde{q}_{2} \in \mathcal{S} N$ such that $\tilde{q}_{2}(s) \cdot \lambda_{2}(s) \leq \lambda_{2}^{\prime}(s)$. Let $q_{1}(s):=4 \tilde{q}_{1} \circ \underline{\kappa}_{1}^{-1} \circ \underline{\alpha}_{1}^{-1}(2 s)$ and $q_{2}(s):=\frac{1}{2} \tilde{q}_{2} \circ$ $\theta_{\lambda_{2}}^{-1}(s)$, where $\theta_{\lambda_{2}}$ is given in Lemma 4.1. We use $q_{1}$ and $q_{2}$ respectively to define $\rho_{1}$ and $\rho_{2}$, and then let $\left(q_{1}, \rho_{1}\right)$ and $\left(q_{2}, \rho_{2}\right)$ respectively generate via Lemma 4.1 new bounding functions $\underline{\alpha}_{1}^{\prime}, \bar{\alpha}_{1}^{\prime}, \alpha_{1}^{\prime}, \sigma_{1}^{\prime}$ and $\underline{\alpha}_{2}^{\prime}, \bar{\alpha}_{2}^{\prime}, \alpha_{2}^{\prime}, \sigma_{2}^{\prime}$.

Let arbitrary strictly positive real numbers $\left(\Delta_{x}, \Delta_{u}, \nu\right)$ be given. Let $\left(\Delta_{x}, \Delta_{u}, \frac{\nu}{2}\right)$ generate via item 1 of the theorem and Lemma 4.1 $T_{1}^{*}$ and $\rho_{1}\left(V_{1 T}\right)$ and let $\left(\gamma_{2}\left(\Delta_{x}\right), \gamma_{3}\left(\Delta_{u}\right), \frac{\nu}{2}\right)$ generate via item 2 of the theorem and Lemma $4.1 T_{2}^{*}$ and $\rho_{2}\left(V_{2 T}\right)$. Let $T^{*}:=\min \left\{T_{1}^{*}, T_{2}^{*}\right\}$. We now define $V_{T}$ as (17). Let $w_{x}(x):=w_{x_{1}}(x)$ and $w_{u}(u):=w_{u_{1}}(u)$. In all calculations below we consider arbitrary $\left|w_{x}(x)\right| \leq \Delta_{x},\left|w_{u}(u)\right| \leq \Delta_{u}$ and $T \in\left(0, T^{*}\right)$. Note that this implies $\left|w_{x_{2}}(x)\right| \leq \gamma_{2}\left(\Delta_{x}\right)$ and $\left|w_{u_{2}}(x)\right| \leq \gamma_{3}\left(\Delta_{u}\right)$.

It follows from the definition of $V_{T}$ that

$$
\begin{gathered}
\rho_{1} \circ \underline{\alpha}_{1}\left(\left|w_{\underline{\alpha}_{1}}(x)\right|\right)+\rho_{2} \circ \underline{\alpha}_{2}\left(\left|w_{\underline{\alpha}_{2}}(x)\right|\right) \leq V_{T}(x) \\
\leq \rho_{1} \circ \bar{\alpha}_{1}\left(\left|w_{\bar{\alpha}_{1}}(x)\right|\right)+\rho_{2} \circ \bar{\alpha}_{2}\left(\left|w_{\bar{\alpha}_{2}}(x)\right|\right) .
\end{gathered}
$$

Then by Remark A.1, there exist $\underline{\alpha}, \bar{\alpha} \in \mathcal{K}_{\infty}$ such that (A.15) holds. Using condition 3 of the theorem and the definition of $\lambda_{2}^{\prime}$, we have:

$$
\begin{aligned}
& V_{T}\left(F_{T}\right)-V_{T}(x) \\
& =\rho_{1}\left(V_{1 T}\left(F_{T}\right)\right)-\rho_{1}\left(V_{1 T}\right)+\rho_{2}\left(V_{2 T}\left(F_{T}\right)\right)-\rho_{2}\left(V_{2 T}\right) \\
& \leq T\left[-\alpha_{1}^{\prime}\left(\left|w_{\alpha_{1}}(x)\right|\right)+\sigma_{1}^{\prime}\left(\left|w_{\sigma_{1}}(u)\right|\right)+\frac{\nu}{2}\right. \\
& \quad-\alpha_{2}^{\prime}\left(\left|w_{\alpha_{2}}(x)\right|\right)+\lambda_{2}^{\prime} \circ \gamma_{1}\left(\left|w_{\alpha_{1}}(x)\right|\right) \\
& \left.\quad+\sigma_{2}^{\prime}\left(\left|w_{\sigma_{2}}(u)\right|\right)+\frac{\nu}{2}\right] \\
& \leq T\left[-\alpha_{2}^{\prime}\left(\left|w_{\alpha_{2}}(x)\right|\right)-\frac{1}{2} \alpha_{1}^{\prime}\left(\left|w_{\alpha_{1}}(x)\right|\right)\right. \\
& \left.\quad+\sigma_{1}^{\prime}\left(\left|w_{\sigma_{1}}(u)\right|\right)+\sigma_{2}^{\prime}\left(\left|w_{\sigma_{2}}(u)\right|\right)+\nu\right]
\end{aligned}
$$

Finally, using Remark A.1, there exist $\sigma \in \mathcal{K}$ and $\alpha \in$ $\mathcal{K}_{\infty}$ that

$$
\begin{aligned}
V_{T}\left(F_{T}\right)-V_{T}(x) \leq T & {\left[\sigma\left(\left|w_{\sigma_{1}}(u)\right|+\left|w_{\sigma_{2}}(u)\right|\right)\right.} \\
& \left.-\alpha\left(\left|w_{\alpha_{1}}(x)\right|+\left|w_{\alpha_{2}}(x)\right|\right)+\nu\right] .
\end{aligned}
$$

This completes the proof of Theorem 4.2. 